\title{
GRAPH-BASED CHANGE-POINT DETECTION
}

\author{
By HaO Chen* and Nancy Zhang ${ }^{\dagger}$ \\ * Department of Statistics, University of California, Davis \\ $\dagger$ Department of Statistics, The Wharton School, University of \\ Pennsylvania
}

\section{Supplementary Materials}

\section{SUPPLEMENT A: PROOFS FOR LEMMAS AND PROPOSITIONS}

A.1. Proof of Lemma 2.1. When observations $\mathbf{y}_{i}$ and $\mathbf{y}_{j}$ are linked in the graph, the edge is denoted as $(i, j)$. Then,

$$
\mathbf{E}\left(R_{G}(t)\right)=\sum_{(i, j) \in G} \mathbf{P}\left(g_{i}(t) \neq g_{j}(t)\right)=p_{1}(t)|G|,
$$

because there are $2 t(n-t)$ ways to place $i$ and $j$ on the two sides of $t$ among all $n(n-1)$ ways.

For the second moment,

$$
\mathbf{E}\left(R_{G}^{2}(t)\right)=\sum_{(i, j),(k, l) \in G} \mathbf{P}\left(g_{i}(t) \neq g_{j}(t), g_{k}(t) \neq g_{l}(t)\right) .
$$

By examining different ways of placing $i, j, k, l$, we have

$$
\begin{aligned}
\mathbf{P}\left(g_{i}(t) \neq\right. & \left.g_{j}(t), g_{k}(t) \neq g_{l}(t)\right) \\
& = \begin{cases}\frac{2 t(n-t)}{n(n-1)}=p_{1}(t) & \text { if }\left\{\begin{array}{l}
i=k, j=l \\
i=l, j=k
\end{array}\right. \\
\frac{t(n-t)}{n(n-1)}=\frac{1}{2} p_{1}(t) & \text { if }\left\{\begin{array}{l}
i=k, j \neq l \\
i=l, j \neq k \\
j=k, i \neq l \\
j=l, i \neq k
\end{array}\right. \\
\frac{4 t(t-1)(n-t)(n-t-1)}{n(n-1)(n-2)(n-3)}=p_{2}(t) & \text { if } i, j, k, l \text { all different. }\end{cases}
\end{aligned}
$$

So

$$
\begin{aligned}
\mathbf{E}\left(R_{G}^{2}(t)\right) & =\sum_{(i, j) \in G} p_{1}(t)+\sum_{\substack{(i, j),(i, k) \in G \\
j \neq k}} \frac{1}{2} p_{1}(t)+\sum_{\substack{(i, j),(k, l) \in G \\
i, j, k, l \text { all different }}} p_{2}(t) \\
& =p_{2}(t)|G|+\left(\frac{1}{2} p_{1}(t)-p_{2}(t)\right) \sum_{i}\left|G_{i}\right|^{2}+p_{2}(t)|G|^{2} .
\end{aligned}
$$

$\operatorname{Var}\left(R_{G}(t)\right)$ follows from $\mathbf{E}\left(R_{G}^{2}(t)\right)-\mathbf{E}^{2}\left(R_{G}(t)\right)$. 
A.2. Proof of Theorem 3.1. We here prove that $\left\{Z_{G}([n u]): 0<\right.$ $u<1\}$ converges to a Gaussian process. The proof for the convergence of $\left\{Z_{G}([n u],[n v]): 0<u<v<1\right\}$ to two-dimensional Gaussian random field can be done in the same manner but with a more careful treatment of the indices.

To prove $\left\{Z_{G}([n u]): 0<u<1\right\}$ converges to a Gaussian process, we only need to show that $\left(Z_{G}\left(\left[n u_{1}\right]\right), Z_{G}\left(\left[n u_{2}\right]\right), \ldots, Z_{G}\left(\left[n u_{K}\right]\right)\right)$ becomes multivariate Gaussian as $n \rightarrow \infty$ for any $0<u_{1}<u_{2}<\cdots<u_{K}<1$ and fixed K. For simplicity, let $t_{k}=\left[n u_{k}\right], k=1, \ldots, K$.

To prove that $\left(Z_{G}\left(t_{1}\right), Z_{G}\left(t_{2}\right), \ldots, Z_{G}\left(t_{K}\right)\right)$ is multivariate Gaussian, we take one step back. In permutation distribution, we permute the order of the observations. Let $\pi(i)$ be the observed time of $\mathbf{y}_{i}$ after permutation, then $(\pi(1), \ldots, \pi(n))$ is a permutation of $1, \ldots, n$. On the other hand, to obtain the permutation distribution, we can do it in two steps: 1) For each $i, \pi(i)$ is sampled uniformly from 1 to $n ; 2)$ only those that each value in $\{1, \ldots, n\}$ is sampled once are retained. It is easy to see that each permutation has the same occurrence probability after these two steps.

We call the distribution resulting from only performing the first step the bootstrap distribution, and we use $\mathbf{P}_{\mathrm{B}}, \mathbf{E}_{\mathrm{B}}$ and $\mathbf{V a r}_{\mathrm{B}}$ to denote the probability, expectation and variance, respectively. (P, E, Var without the subscript $\mathrm{B}$ are used to denote the equivalences under the permutation distribution.) Let

$$
\begin{aligned}
Z_{G}^{B}(t) & =-\frac{R_{G}(t)-\mathbf{E}_{\mathrm{B}}\left(R_{G}(t)\right)}{\sqrt{\operatorname{Var}_{\mathrm{B}}\left(R_{G}(t)\right)}}, \\
X^{B}(t) & =\frac{n^{B}(t)-t}{\sqrt{t(1-t / n)}}, \text { where } n^{B}(t)=\sum_{i=1}^{n} I_{\pi(i) \leq t} .
\end{aligned}
$$

Then following a similar argument in the proof for Lemma 2.1 but replacing the permutation distribution with bootstrap distribution, we have

$$
\begin{aligned}
\mathbf{E}_{\mathrm{B}}\left(R_{G}(t)\right) & =p_{1}^{B}(t)|G| \\
\operatorname{Var}_{\mathrm{B}}\left(R_{G}(t)\right) & =p_{2}^{B}(t)|G|+\left(\frac{1}{2} p_{1}^{B}(t)-p_{2}^{B}(t)\right) \sum_{i}\left|G_{i}\right|^{2}
\end{aligned}
$$

where

$$
p_{1}^{B}(t)=\frac{2 t(n-t)}{n^{2}}, \quad p_{2}^{B}(t)=\frac{4 t^{2}(n-t)^{2}}{n^{4}} .
$$

We prove the following two lemmas. 
Lemma A.1. Under conditions 1 and 2, for $0<u_{1}<u_{2}<\cdots<u_{k}<1$, as $n \rightarrow \infty$, under the bootstrap distribution,

$$
\left(Z_{G}^{B}\left(t_{1}\right), Z_{G}^{B}\left(t_{2}\right), \ldots, Z_{G}^{B}\left(t_{K}\right), X^{B}\left(t_{1}\right), X^{B}\left(t_{2}\right), \ldots, X^{B}\left(t_{K}\right)\right)
$$

is multivariate normal and the covariance matrix of

$$
\left(X^{B}\left(t_{1}\right), X^{B}\left(t_{2}\right), \ldots, X^{B}\left(t_{K}\right)\right)
$$

is positive definite.

LemmA A.2. When $|G| \sim o\left(n^{2}\right)$, for $t \sim \mathcal{O}(n)$, as $|G| \rightarrow \infty$, we have

1.

$$
\frac{\operatorname{Var}_{\mathrm{B}}\left(R_{G}(t)\right)}{\operatorname{Var}\left(R_{G}(t)\right)} \rightarrow 1
$$

2.

$$
\frac{\mathbf{E}_{\mathrm{B}}\left(R_{G}(t)\right)-\mathbf{E}\left(R_{G}(t)\right)}{\sqrt{\operatorname{Var}_{\mathrm{B}}\left(R_{G}(t)\right)}} \rightarrow 0 .
$$

From Lemma A.1, $\left(Z_{G}^{B}\left(t_{1}\right), Z_{G}^{B}\left(t_{2}\right), \ldots, Z_{G}^{B}\left(t_{K}\right) \mid X^{B}\left(t_{1}\right), X^{B}\left(t_{2}\right), \ldots, X^{B}\left(t_{K}\right)\right.$ is multivariate normal under the bootstrap distribution. Since $\left(Z_{G}^{B}\left(t_{1}\right), Z_{G}^{B}\left(t_{2}\right)\right.$, $\left.\ldots, Z_{G}^{B}\left(t_{K}\right) \mid X^{B}\left(t_{1}\right)=0, X^{B}\left(t_{2}\right)=0, \ldots, X^{B}\left(t_{K}\right)=0\right)$ under the bootstrap distribution has the same distribution as $\left(Z_{G}^{B}\left(t_{1}\right), Z^{B}\left(t_{1}\right), \ldots, Z^{B}\left(t_{K}\right)\right)$ under the permutation distribution, and notice that

$$
Z_{G}(t)=\frac{\operatorname{Var}_{\mathrm{B}}\left(R_{G}(t)\right)}{\operatorname{Var}\left(R_{G}(t)\right)}\left(Z_{G}^{B}(t)-\frac{\mathbf{E}_{\mathrm{B}}\left(R_{G}(t)\right)-\mathbf{E}\left(R_{G}(t)\right)}{\sqrt{\operatorname{Var}_{\mathrm{B}}\left(R_{G}(t)\right)}}\right),
$$

we conclude that $\left(Z_{G}\left(\left[n u_{1}\right]\right), Z_{G}\left(\left[n u_{2}\right]\right), \ldots, Z_{G}\left(\left[n u_{K}\right]\right)\right)$ is multivariate Gaussian under the permutation distribution.

We next prove the two lemmas.

Proof for Lemma A.1. To show that (A.1) is multivariate normal, we only need to show that $\sum_{k=1}^{K}\left(a_{k} Z_{G}^{B}\left(t_{k}\right)+b_{k} X^{B}\left(t_{k}\right)\right)$ is normal for any fixed $\left\{a_{k}\right\}$ and $\left\{b_{k}\right\}$.

If $\operatorname{Var}_{\mathrm{B}}\left(\sum_{k=1}^{K}\left(a_{k} Z_{G}^{B}\left(t_{k}\right)+b_{k} X^{B}\left(t_{k}\right)\right)\right)=0, \sum_{k=1}^{K}\left(a_{k} Z_{G}^{B}\left(t_{k}\right)+b_{k} X^{B}\left(t_{k}\right)\right)$ is degenerating and we can claim any distribution for it. For non-degenerating case, let $\left.\sigma_{0}^{2}:=\operatorname{Var}_{\mathrm{B}}\left(a_{k} Z_{G}^{B}\left(t_{k}\right)+b_{k} X^{B}\left(t_{k}\right)\right)\right)$. Then $\sigma_{0} \sim \mathcal{O}(1)$. We prove the Gaussianity of $\sum_{k=1}^{K}\left(a_{k} Z_{G}^{B}\left(t_{k}\right)+b_{k} X^{B}\left(t_{k}\right)\right)$ by the Stein's method.

Consider sums of the form $W=\sum_{i \in \mathcal{J}} \xi_{i}$, where $\mathcal{J}$ is an index set and $\xi$ are random variables with $E\left[\xi_{i}\right]=0$, and $E\left[W^{2}\right]=1$. The following assumption restricts the dependence between $\left\{\xi_{i}: i \in \mathcal{J}\right\}$. 
ASsumption A.3. (Chen and Shao, 2005, p. 17) For each $i \in \mathcal{J}$ there exists $S_{i} \subset T_{i} \subset \mathcal{J}$ such that $\xi_{i}$ is independent of $\xi_{S_{i}^{c}}$ and $\xi_{S_{i}}$ is independent of $\xi_{T_{i}^{c}}$.

We will use the following specific form of Stein's method.

Theorem A.4. (Chen and Shao, 2005, Theorem 3.4) Under Assumption A.3, we have

$$
\sup _{h \in \operatorname{Lip}(1)}|\mathbf{E} h(W)-\mathbf{E} h(Z)| \leq \delta
$$

where $\operatorname{Lip}(1)=\{h: \mathbb{R} \rightarrow \mathbb{R}\}, Z$ has $\mathcal{N}(0,1)$ distribution and

$$
\delta=2 \sum_{i \in \mathcal{J}}\left(\mathbf{E}\left|\xi_{i} \eta_{i} \theta_{i}\right|+\left|\mathbf{E}\left(\xi_{i} \eta_{i}\right)\right| \mathbf{E}\left|\theta_{i}\right|\right)+\sum_{i \in \mathcal{J}} \mathbf{E}\left|\xi_{i} \eta_{i}^{2}\right|
$$

with $\eta_{i}=\sum_{j \in S_{i}} \xi_{j}$ and $\theta_{i}=\sum_{j \in T_{i}} \xi_{j}$, where $S_{i}$ and $T_{i}$ are defined in Assumption A.3.

We adopt the same notation with the index set $\mathcal{J}=\{G, 1, \ldots, n\}$.

Let

$$
\xi_{e, k}=\frac{I_{g_{\pi_{\left(e_{-}\right)}}\left(t_{k}\right) \neq g_{\pi\left(e_{+}\right)}\left(t_{k}\right)}-p_{1}^{B}\left(t_{k}\right)}{\sigma^{B}\left(t_{k}\right)}
$$

Since $I_{g_{\pi\left(e_{-}\right)}\left(t_{k}\right) \neq g_{\pi\left(e_{+}\right)}\left(t_{k}\right)} \in\{0,1\}, p_{1}^{B}\left(t_{k}\right) \in(0,1)$, we have

$$
\left|\xi_{e, k}\right| \leq \frac{1}{\sigma^{B}\left(t_{k}\right)}
$$

Let

$$
\xi_{i, k}=\frac{I_{\pi(i) \leq t_{k}}-u_{k}}{\sqrt{n u_{k}\left(1-u_{k}\right)}} .
$$

Similarly, we have

$$
\left|\xi_{i, k}\right| \leq \frac{1}{\sqrt{n u_{k}\left(1-u_{k}\right)}} .
$$

Let $\xi_{e}=\sum_{k} a_{k} \xi_{e, k} / \sigma_{0}, \xi_{i}=\sum_{k} b_{k} \xi_{i, k} / \sigma_{0}$, then $W=\sum_{j \in \mathcal{J}} \xi_{j}=\sum_{k}\left(a_{k} Z_{G}^{B}\left(t_{k}\right)+\right.$ $\left.b_{k} X^{B}\left(t_{k}\right)\right) / \sigma_{0}, \mathbf{E}_{\mathrm{B}}(W)=0, \mathbf{E}_{\mathrm{B}}\left(W^{2}\right)=1$. Let $a=\max \left(\max _{k} a_{k}, \max _{k} b_{k}\right)$, $\sigma=\min \left(\min _{k} \sigma^{B}\left(t_{k}\right), \min _{k} \sqrt{n u_{k}\left(1-u_{k}\right)}\right)$. Then

$$
\left|\xi_{j}\right| \leq \frac{a K}{\sigma \sigma_{0}}, \quad \forall j \in \mathcal{J}
$$


For $e \in G$, let

$$
\begin{aligned}
& S_{e}=\left\{A_{e}, e^{-}, e^{+}\right\}, \\
& T_{e}=B_{e} \cup\left\{\text { Nodes in } A_{e}\right\},
\end{aligned}
$$

where $A_{e}, B_{e}$ defined in (3.3) and (3.4). Then $S_{e}$ and $T_{e}$ satisfy Assumption A.3.

For $i=1, \ldots, n$, let

$$
\begin{aligned}
S_{i} & =G_{i} \\
T_{i} & =G_{i, 2} \cup\left\{\text { Nodes in } G_{i}\right\},
\end{aligned}
$$

where $G_{i, 2}$ is the subgraph of $G$ including all edges connect to $G_{i}$. Then $S_{i}$ and $T_{i}$ satisfy Assumption A.3.

We have $\left|S_{e}\right|=\left|A_{e}\right|+2,\left|T_{e}\right|=\left|B_{e}\right|+\left|A_{e}\right|+1,\left|S_{i}\right|=\left|G_{i}\right|,\left|T_{i}\right|=$ $\left|G_{i, 2}\right|+\left|G_{i}\right|+1$.

By Theorem A.4, we have $\sup _{h \in L i p(1)}|\mathbf{E} h(W)-\mathbf{E} h(Z)| \leq \delta$ for $Z \sim$ $\mathcal{N}(0,1)$, where

$$
\begin{aligned}
\delta= & 2 \sum_{j \in \mathcal{J}}\left(\mathbf{E}\left|\xi_{j} \eta_{j} \theta_{j}\right|+\left|\mathbf{E}\left(\xi_{j} \eta_{j}\right)\right| \mathbf{E}\left|\theta_{j}\right|\right)+\sum_{j \in \mathcal{J}} \mathbf{E}\left|\xi_{j} \eta_{j}^{2}\right| \\
= & 2 \sum_{e \in G}\left(\mathbf{E}\left|\xi_{e} \eta_{e} \theta_{e}\right|+\left|\mathbf{E}\left(\xi_{e} \eta_{e}\right)\right| \mathbf{E}\left|\theta_{e}\right|\right)+\sum_{e \in G} \mathbf{E}\left|\xi_{e} \eta_{e}^{2}\right| \\
& \quad+2 \sum_{i=1}^{n}\left(\mathbf{E}\left|\xi_{i} \eta_{i} \theta_{i}\right|+\left|\mathbf{E}\left(\xi_{i} \eta_{i}\right)\right| \mathbf{E}\left|\theta_{i}\right|\right)+\sum_{i=1}^{n} \mathbf{E}\left|\xi_{i} \eta_{i}^{2}\right| \\
\leq & \frac{a^{3} K^{3}}{\sigma^{3} \sigma_{0}^{3}}\left(\sum_{e \in G} 5\left(\left|A_{e}\right|+2\right)\left(\left|B_{e}\right|+\left|A_{e}\right|+1\right)+\sum_{i=1}^{n} 5\left|G_{i}\right|\left(\left|G_{i, 2}\right|+\left|G_{i}\right|+1\right)\right) \\
\leq & \frac{a^{3} K^{3}}{\sigma^{3} \sigma_{0}^{3}}\left(45 \sum_{e \in G}\left|A_{e}\right|\left|B_{e}\right|+15 \sum_{i=1}^{n}\left|G_{i}\right|\left|G_{i, 2}\right|\right)
\end{aligned}
$$

Observe that if $e=(i, j)$, then $G_{i}, G_{j} \subseteq A_{e}, G_{i, 2}, G_{j, 2} \subseteq B_{e}$. For each node $\mathbf{y}_{i}$, we can randomly pick an edge $e$ that connects node $\mathbf{y}_{i}$, and we have $\left|G_{i}\right|\left|G_{i, 2}\right| \leq\left|A_{e}\right|\left|B_{e}\right|$. Each node in the graph can be picked twice in maximum since an edge connects two nodes, therefore,

$$
\sum_{i=1}^{n}\left|G_{i}\right|\left|G_{i, 2}\right| \leq 2 \sum_{e \in G}\left|A_{e}\right|\left|B_{e}\right| .
$$

So

$$
\delta \leq \frac{75 a^{3} K^{3}}{\sigma^{3} \sigma_{0}^{3}} \sum_{e \in G}\left|A_{e}\right|\left|B_{e}\right| .
$$


Notice that $\sigma \sim \mathcal{O}\left(\min \left(n^{1 / 2},|G|^{1 / 2}\right)\right)$. When $|G| \sim \mathcal{O}\left(n^{\alpha}\right)$, we have $\sigma \sim$ $\mathcal{O}\left(n^{0.5(\alpha \wedge 1)}\right)$. When $\sum_{e \in G}\left|A_{e}\right|\left|B_{e}\right| \sim o\left(n^{1.5(\alpha \wedge 1)}\right)$, we have $\delta \rightarrow 0$ as $n \rightarrow \infty$.

Let $\Sigma_{X}$ be the covariance matrix of $\left(X^{B}\left(t_{1}\right), X^{B}\left(t_{2}\right), \ldots, X^{B}\left(t_{K}\right)\right)$. It's not hard to derive that for $i \leq j$,

$$
\Sigma_{X}(i, j)=\Sigma_{X}(j, i)=\frac{t_{i}\left(1-t_{j} / n\right)}{\sqrt{t_{i}\left(1-t_{i} / n\right) t_{j}\left(1-t_{j} / n\right)}},
$$

where $t_{K+1} \triangleq n$.

$\Sigma_{X}^{-1}$ admits the Cholesky decomposition

$$
\Sigma_{X}^{-1}=L L^{\prime}
$$

where

$$
L(i, j)= \begin{cases}\frac{\sqrt{1-t_{i} / n}}{\sqrt{1-t_{i} / t_{i+1}}}, & j=i \\ -\frac{t_{i} \sqrt{1-t_{i} / n}}{t_{i+1} \sqrt{1-t_{i} / t_{i+1}}}, & j=i-1 \\ 0, & \text { otherwise }\end{cases}
$$

Therefore,

$$
\left|\Sigma_{X}\right|=\frac{\prod_{k=1}^{K}\left(1-t_{k} / t_{k+1}\right)}{\prod_{k=1}^{K}\left(1-t_{k} / n\right)}
$$

is positive definite.

Proof for Lemma A.2. Let $u=\lim _{n \rightarrow \infty} t / n$, then

$$
\begin{aligned}
\lim _{n \rightarrow \infty} p_{1}(t) & =\lim _{n \rightarrow \infty} p_{1}^{B}(t)=2 u(1-u), \\
\lim _{n \rightarrow \infty} p_{2}(t) & =\lim _{n \rightarrow \infty} p_{2}^{B}(t)=4 u^{2}(1-u)^{2}, \\
\lim _{n \rightarrow \infty} \operatorname{Var}\left(R_{G}(t)\right) & =\lim _{n \rightarrow \infty} \operatorname{Var}_{\mathrm{B}}\left(R_{G}(t)\right)=4 u^{2}(1-u)^{2}|G|+u(1-u)(1-2 u)^{2} \sum_{i}\left|G_{i}\right|^{2} .
\end{aligned}
$$

So

$$
\frac{\operatorname{Var}_{\mathrm{B}}\left(R_{G}(t)\right)}{\operatorname{Var}\left(R_{G}(t)\right)} \rightarrow 1
$$

Since

$$
\mathbf{E}_{\mathrm{B}}\left(R_{G}(t)\right)-\mathbf{E}\left(R_{G}(t)\right)=\left(p_{1}^{B}(t)-p_{1}(t)\right)|G|=-\frac{2 t(n-t)}{n^{3}}|G|,
$$


we have

$$
\begin{aligned}
& \lim _{n \rightarrow \infty} \frac{\mathbf{E}_{\mathrm{B}}\left(R_{G}(t)\right)-\mathbf{E}\left(R_{G}(t)\right)}{\sqrt{\operatorname{Var}_{\mathrm{B}}\left(R_{G}(t)\right)}}=-\lim _{n \rightarrow \infty} \frac{2 u(1-u)|G| / n}{\sqrt{4 u^{2}(1-u)^{2}|G|+u(1-u)(1-2 u)^{2} \sum_{i}\left|G_{i}\right|^{2}}} \\
&=-\lim _{n \rightarrow \infty} \frac{2 u(1-u)}{\sqrt{4 u^{2}(1-u)^{2} n^{2} /|G|+u(1-u)(1-2 u)^{2} n^{2} \sum_{i}\left|G_{i}\right|^{2} /|G|^{2}}}
\end{aligned}
$$

which is 0 when $|G| \sim o\left(n^{2}\right)$.

A.3. Proof of Lemma 3.3. First observe that $\rho_{G}^{\star}(u, u)=1$, which holds for (3.5) Because of the interchangeability of $u$ and $v$ in the definition of $\rho_{G}(u, v)$, it is enough to show that when $u<v$,

$$
\rho_{G}^{\star}(u, v)=\frac{\left.2 u^{2}(1-v)^{2}|G|+u(1-v)\right)(1-2 u)(1-2 v) \sum_{i}\left|G_{i}\right|^{2}}{\sigma_{G}^{\star}(u) \sigma_{G}^{\star}(v)} .
$$

Let $\rho_{G, n}(u, v) \triangleq \operatorname{cov}\left(Z_{G}([n u]), Z_{G}([n v])\right)$, then $\rho_{G}(u, v)=\lim _{n \rightarrow \infty} \rho_{G, n}(u, v)$. Let $s=[n u], t=[n v]$, then $s<t$, and $\lim _{n \rightarrow \infty} s / n=u, \lim _{n \rightarrow \infty} t / n=v$. Since

$$
\operatorname{cov}\left(Z_{G}(s), Z_{G}(t)\right)=\frac{\mathbf{E}\left(R_{G}(s) R_{G}(t)\right)-\mathbf{E}\left(R_{G}(s)\right) \mathbf{E}\left(R_{G}(t)\right)}{\sqrt{\operatorname{Var}\left(R_{G}(s)\right) \operatorname{Var}\left(R_{G}(t)\right)}},
$$

where the expressions for $\mathbf{E}\left(R_{G}(s)\right), \mathbf{E}\left(R_{G}(t)\right), \operatorname{Var}\left(R_{G}(s)\right), \operatorname{Var}\left(R_{G}(t)\right)$ can be found in Lemma 2.1, we only need to figure out

$$
\mathbf{E}\left(R_{G}(s) R_{G}(t)\right)=\sum_{(i, j),(k, l) \in G} \mathbf{P}\left(g_{i}(s) \neq g_{j}(s), g_{k}(t) \neq g_{l}(t)\right) .
$$

By examining different ways of placing $i, j, k, l$, we have

$$
\begin{aligned}
& \mathbf{P}\left[g_{i}(s) \neq g_{j}(s), g_{k}(t) \neq g_{l}(t)\right] \\
& \quad= \begin{cases}\frac{2 s(n-t)}{n(n-1)}:=q_{1}(s, t) & \text { if }\left\{\begin{array}{l}
i=k, j=l \\
i=l, j=k
\end{array}\right. \\
\frac{s(n-t)(n+2 t-2 s-2)}{n(n-1)(n-2)}:=q_{2}(s, t) & \text { if }\left\{\begin{array}{l}
i=k, j \neq l \\
i=l, j \neq k \\
j=k, i \neq l \\
j=l, i \neq k
\end{array}\right. \\
\frac{4 s(n-t)[(s-1)(n-s-1)+(t-s)(n-s-2)]}{n(n-1)(n-2)(n-3)}:=q_{3}(s, t) & \text { if } i, j, k, l \text { all different. }\end{cases}
\end{aligned}
$$


Then

$$
\begin{aligned}
& \mathbf{E}\left(R_{G}(s) R_{G}(t)\right)=\sum_{\substack{(i, j),(k, l) \in G \\
(i, j) \in G}} \mathbf{P}\left(g_{i}(s) \neq g_{j}(s), g_{k}(t) \neq g_{l}(t)\right) \\
& =\sum_{\substack{(i, j),(i, k) \in G \\
j \neq k}} q_{2}(s, t)+\sum_{\substack{(i, j),(k, l) \in G \\
i, j, k, l \text { all different }}} q_{3}(s, t) \\
& =\left(q_{1}(s, t)-2 q_{2}(s, t)+q_{3}(s, t)\right)|G|+\left(q_{2}(s, t)-q_{3}(s, t)\right) \sum_{i=1}^{n}\left|G_{i}\right|^{2}+q_{3}(s, t)|G|^{2} .
\end{aligned}
$$

So

$$
\begin{aligned}
\lim _{n \rightarrow \infty} \mathbf{E}\left(R_{G}(s) R_{G}(t)\right)= & 4 u^{2}(1-v)^{2}|G|+u(1-v)(1-2 u)(1-2 v) \sum_{i=1}^{n}\left|G_{i}\right|^{2} \\
& +4 u v(1-u)(1-v)|G|^{2} .
\end{aligned}
$$

Together with

$$
\begin{aligned}
\lim _{n \rightarrow \infty} \mathbf{E}\left(R_{G}(s)\right) & =2 u(1-u)|G|, \\
\lim _{n \rightarrow \infty} \operatorname{Var}\left(R_{G}(s)\right) & =4 u^{2}(1-u)^{2}|G|+u(1-u)(1-2 u)^{2} \sum_{i=1}^{n}\left|G_{i}\right|^{2},
\end{aligned}
$$

and similar for $R_{G}(t)$, we have (A.2).

A.4. Proof of Proposition 3.4. We first show the single change-point case. We adopt Woodroofe's method (Woodroofe, 1976, 1978) by condition on the first cross-over.

$$
\mathbf{P}\left(\max _{n_{0} \leq t \leq n_{1}} Z_{G}^{\star}(t / n)>b\right)
$$

$$
=\sum_{n_{0} \leq t \leq n_{1}} \int_{0}^{\infty} \mathbf{P}\left(Z_{G}^{\star}(t / n)=b+d x\right) \mathbf{P}\left(\max _{n_{0} \leq s<t} Z_{G}^{\star}(s / n)<b \mid Z_{G}^{\star}(t / n)=b+d x\right)
$$

By change of measure and rearranging the terms, we have

$$
\begin{aligned}
& \mathbf{P}\left(\max _{n_{0} \leq t \leq n_{1}} Z_{G}^{\star}(t / n)>b\right) \\
& =\frac{\phi(b)}{b} \sum_{n_{0} \leq t \leq n_{1}} \int_{0}^{\infty} e^{-x-\frac{x^{2}}{2 b^{2}}} \mathbf{P}\left(\max _{n_{0} \leq s<t} b\left(Z_{G}^{\star}(s / n)-Z_{G}^{\star}(t / n)\right)<-x \mid Z_{G}^{\star}(t / n)=b+\frac{x}{b}\right) d x .
\end{aligned}
$$


Since $b \rightarrow \infty$, if $x \sim o\left(b^{2}\right)$, then $\frac{x^{2}}{2 b^{2}}$ is negligible to $x$ and $\frac{x}{b}$ is negligible to $b$; while if $x \sim \mathcal{O}(b)$, then $x+\frac{x^{2}}{2 b^{2}} \rightarrow \infty$, and the integrand becomes 0 , so

$$
\begin{aligned}
& \mathbf{P}\left(\max _{n_{0} \leq t \leq n_{1}} Z_{G}^{\star}(t / n)>b\right) \\
& \approx \frac{\phi(b)}{b} \sum_{n_{0} \leq t \leq n_{1}} \int_{0}^{\infty} e^{-x} \mathbf{P}\left(\max _{n_{0} \leq s<t} b\left(Z_{G}^{\star}(s / n)-Z_{G}^{\star}(t / n)\right)<-x \mid Z_{G}^{\star}(t / n)=b\right) d x .
\end{aligned}
$$

Notice that for $u<v$,

$$
b\left(Z_{G}^{\star}(u)-Z_{G}^{\star}(v)\right) \mid\left(Z_{G}^{\star}(v)=b\right) \sim \mathcal{N}\left(\left(\rho_{G}(u, v)-1\right) b^{2},\left(1-\rho_{G}^{2}(u, v)\right) b^{2}\right) .
$$

Let $\delta=v-u$, by Taylor expansion, we have

$$
\begin{aligned}
& \rho_{G}(u, v)=1+f_{v,-}^{\prime}(0) \delta+f_{v,-}^{\prime \prime}(0) \delta^{2} / 2+\mathcal{O}\left(\delta^{3}\right), \\
& \rho_{G}^{2}(u, v)=1+2 f_{v,-}^{\prime}(0) \delta+\left(\left(f_{v,-}^{\prime}\right)^{2}+f_{v,-}^{\prime \prime}(0)\right) \delta^{2}+\mathcal{O}\left(\delta^{3}\right) .
\end{aligned}
$$

So for $\delta \sim \mathcal{O}\left(n^{-1}\right)$,

$$
b\left(Z_{G}^{\star}(u)-Z_{G}^{\star}(v)\right) \mid\left(Z_{G}^{\star}(v)=b\right) \sim \mathcal{N}\left(-f_{v,-}^{\prime}(0)|\delta| b^{2}, 2 f_{v,-}^{\prime}(0)|\delta| b^{2}\right) .
$$

One can show that, for $b=b_{0} \sqrt{n}$, and $n \rightarrow \infty$,

$$
\lim _{k \rightarrow \infty} \limsup _{n \rightarrow \infty} \sum_{|i-t|>k} \mathbf{P}\left(Z_{G}^{\star}(i / n)>b \mid Z_{G}^{\star}(t / n)=b+d x\right)=0 .
$$

Let $W_{m}^{(t)}$ be a random walk with $W_{1}^{(t)} \sim \mathcal{N}\left(\mu^{(t)},\left(\sigma^{(t)}\right)^{2}\right)$, where $\mu^{(t)}=$ $\frac{1}{n} f_{v,-}^{\prime}(0) b^{2},\left(\sigma^{(t)}\right)^{2}=2 \mu^{(t)}$. Then

$$
\begin{aligned}
\mathbf{P}\left(\max _{n_{0} \leq s<t} b\left(Z_{G}^{\star}(s / n)-Z_{G}^{\star}(t / n)\right)<-x \mid Z_{G}^{\star}(t / n)=b\right) & \sim \mathbf{P}\left(\max _{n_{0} \leq s \leq t}-W_{t-s}^{(t)}<-x\right) \\
& \sim \mathbf{P}\left(\min _{m \geq 1} W_{m}^{(t)}>x\right) .
\end{aligned}
$$

Together with the fact

$$
\int_{0}^{\infty} \exp -2 \mu x / \sigma \mathbf{P}\left(\min _{m \geq 1} W_{m}>x\right) d x=\mu \nu(2 \mu / \sigma),
$$

for a random walk $W_{1} \sim \mathcal{N}(\mu, \sigma)$ (see Siegmund (1992)), we have

$$
\lim _{n \rightarrow \infty} \mathbf{P}\left(\max _{n_{0} \leq t \leq n_{1}} Z_{G}^{\star}(t / n)>b\right) \approx \lim _{n \rightarrow \infty} \frac{\phi(b)}{b} \sum_{n_{0} \leq t \leq n_{1}} b_{0}^{2} f_{t / n,-}^{\prime}(0) \nu\left(b_{0} \sqrt{2 f_{t / n,-}^{\prime}(0)}\right)
$$


For $f_{t / n,-}^{\prime}(0)$, we take the derivative of $\rho_{G}^{\star}(u, v)$, and after some tedious calculation, we have

$$
f_{v,-}^{\prime}(0)=\frac{1}{2 v(1-v)}+\frac{2}{4 v(1-v)+(1-2 v)^{2}\left(\sum_{i}\left|G_{i}\right|^{2} /|G|-4|G|\right)}
$$

Putting everything together, we have

$$
\begin{aligned}
\lim _{n \rightarrow \infty} \mathbf{P}\left(\max _{n_{0} \leq t \leq n_{1}} Z_{G}^{\star}(t / n)>b\right) & \approx \lim _{n \rightarrow \infty} \frac{\phi(b)}{b} \sum_{n_{0} \leq t \leq n_{1}} b_{0}^{2} h_{r_{0}, r_{1}}^{*}(t / n) \nu\left(b_{0} \sqrt{2 h_{r_{0}, r_{1}}^{*}(t / n)}\right) \\
& =\frac{\phi(b)}{b} \int_{x_{0}}^{x_{1}} b_{0}^{2} h_{r_{0}, r_{1}}^{*}(x) \nu\left(b_{0} \sqrt{2 h_{r_{0}, r_{1}}^{*}(x)}\right) n d x \\
& =b \phi(b) \int_{x_{0}}^{x_{1}} h_{r_{0}, r_{1}}^{*}(x) \nu\left(b_{0} \sqrt{2 h_{r_{0}, r_{1}}^{*}(x)}\right) d x
\end{aligned}
$$

Now, we show the changed interval case following the method of Siegmund $(1988,1992)$. We omit most of the technical details, which follow these two papers given that

$$
\rho_{G,\left(u_{1}, u_{2}\right)}^{\star}\left(\delta_{1}, \delta_{2}\right) \triangleq \operatorname{cov}\left(Z_{G}^{\star}\left(u_{1}-\delta_{1}, u_{2}-\delta_{2}\right), Z_{G}^{\star}\left(u_{1}, u_{2}\right)\right) .
$$

is differentiable with the derivative being continuous except at $\delta_{1}=0$ and at $\delta_{2}=0$.

A key intermediate form is

$\mathbf{P}\left(\max _{n_{0} \leq t_{2}-t_{1} \leq n_{1}} Z_{G}^{\star}\left(t_{1} / n, t_{2} / n\right)>b\right)$

$$
\approx \frac{\phi(b)}{b} \sum_{n_{0} \leq t_{2}-t_{1} \leq n_{1}} C_{1}\left(t_{1}, t_{2}\right) b^{2} C_{2}\left(t_{1}, t_{2}\right) b^{2} \times \nu\left(\sqrt{2 C_{1}\left(t_{1}, t_{2}\right) b^{2}}\right) \nu\left(\sqrt{2 C_{2}\left(t_{1}, t_{2}\right) b^{2}}\right),
$$

where $C_{1}, C_{2}$ are the partial derivatives

$$
\begin{gathered}
\left.C_{1}\left(n u_{1}, n u_{2}\right) \equiv \frac{1}{n} \frac{\partial_{-} \rho_{G,\left(u_{1}, u_{2}\right)}^{\star}\left(\delta_{1}, 0\right)}{\partial \delta_{1}}\right|_{\delta_{1}=0}=-\left.\frac{1}{n} \frac{\partial_{+} \rho_{G,\left(u_{1}, u_{2}\right)}^{\star}\left(\delta_{1}, 0\right)}{\partial \delta_{1}}\right|_{\delta_{1}=0}, \\
C_{2}\left(n u_{1}, n u_{2}\right) \equiv-\left.\frac{1}{n} \frac{\partial_{+} \rho_{G,\left(u_{1}, u_{2}\right)}^{\star}\left(0, \delta_{2}\right)}{\partial \delta_{2}}\right|_{\delta_{2}=0} \cdot
\end{gathered}
$$

Under the permutation null, the processes derived from perturbation of the left and right end points,

$$
Z_{G}^{\star}\left(\left(t_{1}+k\right) / n, t_{2} / n\right), \quad k=\ldots,-2,-1,0,1,2, \ldots
$$


and

$$
Z_{G}^{\star}\left(t_{1} / n,\left(t_{2}-k\right) / n\right), \quad k=\ldots,-2,-1,0,1,2, \ldots,
$$

are identical in distribution to the process

$$
Z_{G}^{\star}\left(\left(t_{2}-t_{1}-k\right) / n\right), \quad k=\ldots,-2,-1,0,1,2, \ldots,
$$

Thus, the partial derivatives are equal to the derivative in the one changepoint scenario,

$$
C_{1}\left(t_{1}, t_{2}\right)=C_{2}\left(t_{1}, t_{2}\right)=\frac{1}{n} f_{u_{2}-u_{1},-}^{\prime}(0)
$$

Substituting $\frac{1}{n} f_{u_{2}-u_{1},-}^{\prime}(0)$ for $C_{1}\left(t_{1}, t_{2}\right)$ and $C_{2}\left(t_{1}, t_{2}\right)$ in (A.5) and the double summation goes to an integral as $n \rightarrow \infty$ yields (3.9).

\section{SUPPLEMENT B: SKEWNESS CORRECTION}

B.1. Derivation of (3.13) and (3.15). We first show how to approximate the marginal probability $\mathbf{P}\left(Z_{G}(t) \in b+d x / b\right)$ better by incorporating skewness. In the derivation below we suppress the dependence on the graph $G$ and the time parameter $t$. Consider the probability measure $d \mathbf{Q}_{\theta}=e^{\theta Z-\psi(\theta)} d \mathbf{P}$, where $\psi(\theta)=\log \mathbf{E}_{\mathbf{P}}\left(e^{\theta Z}\right)$. Choose $\theta_{b}$ such that $\dot{\psi}\left(\theta_{b}\right)=$ $\mathbf{E}_{\mathbf{Q}_{\theta_{b}}}(Z)=b$. Then,

$$
\mathbf{P}(Z \in b+d x / b)=\mathbf{E}_{\mathbf{P}}\left(\mathbf{1}_{Z \in b+d x / b}\right) \approx e^{-\theta_{b}(b+x / b)+\psi\left(\theta_{b}\right)} \mathbf{Q}_{\theta_{b}}(Z \in b+d x / b) .
$$

Since under $\mathbf{Q}_{\theta_{b}}, Z$ is centered at $b$ with variance $\ddot{\psi}\left(\theta_{b}\right), \mathbf{Q}_{\theta_{b}}(Z \in b+d x / b)$ can be approximated by the normal density,

$$
\mathbf{Q}_{\theta_{b}}(Z \in b+d x / b) \approx \frac{1}{\sqrt{2 \pi \ddot{\psi}\left(\theta_{b}\right)}} \exp \left(-\frac{x^{2}}{2 b^{2} \ddot{\psi}\left(\theta_{b}\right)}\right) \approx \frac{1}{\sqrt{2 \pi \ddot{\psi}\left(\theta_{b}\right)}} .
$$

The second approximation above is accurate for $x / b \rightarrow 0$.

To obtain $\psi\left(\theta_{b}\right)$ and $\ddot{\psi}\left(\theta_{b}\right)$, we use Taylor expansions, noting that $\psi(0)=$ $\dot{\psi}(0)=0, \ddot{\psi}(0)=1, \dddot{\psi}(0)=\mathbf{E}_{\mathbf{P}}\left(Z^{3}\right) \triangleq \gamma$ :

$$
\begin{aligned}
& \psi(\theta) \approx \psi(0)+\dot{\psi}(0) \theta+\ddot{\psi}(0) \frac{\theta^{2}}{2}+\dddot{\psi}(0) \frac{\theta^{3}}{6}=\frac{\theta^{2}}{2}\left(1+\frac{\gamma \theta}{3}\right), \\
& \ddot{\psi}(\theta) \approx \ddot{\psi}(0)+\ddot{\psi}(0) \theta=1+\gamma \theta .
\end{aligned}
$$


Combining (B.1), (B.2),(B.3) and (B.4) gives

$$
\mathbf{P}(Z \in b+d x / b) \approx \frac{1}{\sqrt{2 \pi\left(1+\gamma \theta_{b}\right)}} \exp \left(-\theta_{b} b-x \theta_{b} / b+\theta_{b}^{2}\left(1+\gamma \theta_{b} / 3\right) / 2\right) .
$$

For an approximation of $\theta_{b}$, we solve $\dot{\psi}\left(\theta_{b}\right)$ by approximating $\psi$ up to the third order,

$$
b=\dot{\psi}\left(\theta_{b}\right) \approx \dot{\psi}(0)+\ddot{\psi}(0) \theta_{b}+\dddot{\psi}(0) \frac{\theta_{b}^{2}}{2}=\theta_{b}+\frac{1}{2} \gamma \theta_{b}^{2},
$$

yielding

$$
\theta_{b} \approx(-1+\sqrt{1+2 \gamma b}) / \gamma
$$

Note that when $\gamma=0, \theta_{b}=b$. (3.13) follows by using (B.5) in (A.3) in the proof of Theorem 3.4 and approximating the $\theta_{b} x / b$ term in the exponent by $x$.

The derivation for (3.15) is similar but to give a better approximation to $\mathbf{P}\left(Z_{G}\left(t_{1}, t_{2}\right) \in b+d x / b\right)$ by incorporating skewness.

B.2. Effect of Skewness and Extrapolation at Boundaries. To gain a better understanding of the role of skewness, we explore the following quantities involved in the $p$-value approximations:

- $\gamma_{G}(t) \triangleq \mathbf{E}\left[Z_{G}^{3}(t)\right]$,

- $\theta_{b, G}(t) \triangleq\left(-1+\sqrt{1+2 \gamma_{G}(t) b}\right) / \gamma_{G}(t)$,

- $S_{G}(t) \triangleq \frac{1}{\sqrt{1+\gamma_{G}(t) \theta_{b, G}(t)}} \exp \left(\frac{1}{2}\left(b-\theta_{b, G}(t)\right)^{2}+\frac{\gamma_{G}(t) \theta_{b, G}(t)^{3}}{6}\right)$.

Figure 1 shows the three quantities versus $t$ for the single change-point scan statistic on a MDP graph when $n=1000, b=3$. Since the structure of MDP is always the same and does not depend on the distribution of $\mathbf{y}_{i}$, Figure 1 is representative of all MDP graphs with $n=1000$ subjects and threshold $b=3$. We can see from the figure that $\gamma$ is always larger than 0 , indicating right skewness. When $\gamma=0, \theta_{b}=b$; when $\gamma>0, \theta_{b}<b$. When $Z_{G}(t)$ is right-skewed, the analytic approximation of the $p$-value assuming Gaussianity is smaller than the actual $p$-value, so the skewness correction should increase the $p$-value approximation. This is indeed true as $S_{G}(t)$ is U-shaped with a minimum of 1 .

Each node in the MDP has degree 1. The shapes of $\gamma_{G}(t)$ and $\theta_{b, G}(t)$ for $Z_{G}(t)$ computed on graphs with very low number of hubs are similar 
to their shapes for $Z_{G}(t)$ computed on the MDP. For example, for data in low dimensions $(<5)$, scans based on MST and NNG constructed based on Euclidean distance have similar skewness properties as described above. However, as the dimension of the data increases, MST and NNG constructed based on Euclidean distance tend to become dominated by hubs, and the distribution of $Z_{G}(t)$ becomes left-skewed. For a left-skewed distribution, $\gamma \leq 0, \theta_{b} \geq b$, and $S \leq 1$. One problem for left-skewed distributions is that if $\gamma$ is smaller than $-1 /(2 b)$, the current approximation does not yield real-valued solution for $\theta_{b}$. This issue is discussed in Remark 3.7 and here we provide a heuristic solution to this problem based on an extrapolation procedure.

We illustrate procedure through a MST constructed on a simulated 100dimensional data based on Euclidean distance. From Figure 2, we see that $\theta_{b, G}(t)$ and $S_{G}(t)$ are not defined except in the middle region. In this case, the integrand

$$
S_{G}(n u) h_{G}(n, x) \nu \sqrt{2 b_{0}^{2} h_{G}(n, x)}
$$

is directly extrapolated to the edge regions using the boundary tangent at each side. If extrapolation is negative, it is set to zero. Figure 3 illustrates the integrand before and after extrapolation.
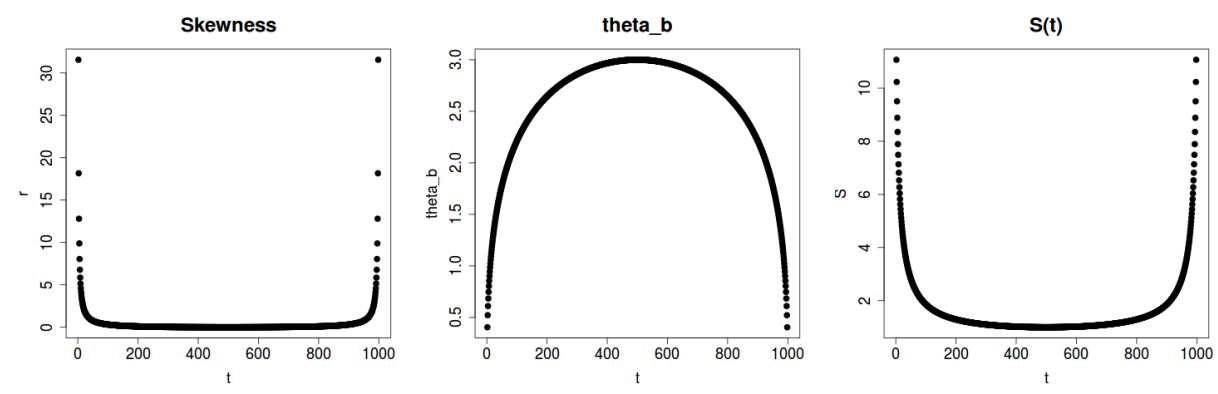

FIG 1. The three quantities, $\gamma_{G}(t), \theta_{b, G}(t)$ and $S_{G}(t)$ from left to right, for a MDP graph. $n=1000, b=3$.

\section{SUPPLEMENT C: CHECKING ANALYTIC APPROXIMATIONS TO $P$-VALUES}

\section{C.1. Critical Value.}

C.1.1. A Single Change-Point Alternative (NNG). Tables 1 - 2 show the results of $p$-value approximations for the single change-point alternative with 

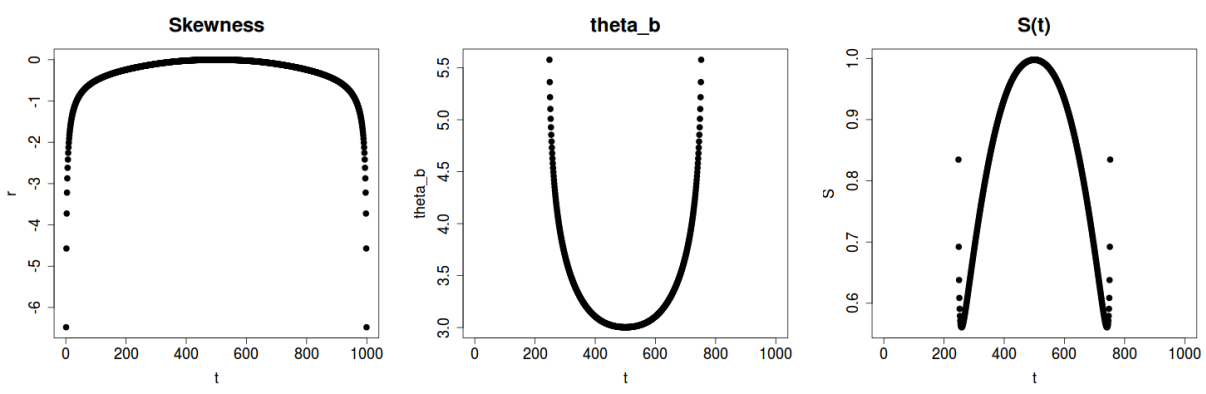

FIG 2. The three quantities, $\gamma_{G}(t), \theta_{b, G}(t)$ and $S_{G}(t)$ from left to right, for a MST graph constructed using Euclidean distance on a sequence of $n=1000$ observations iid drawn from $N\left(\mathbf{0}, I_{100}\right) . b=3$.
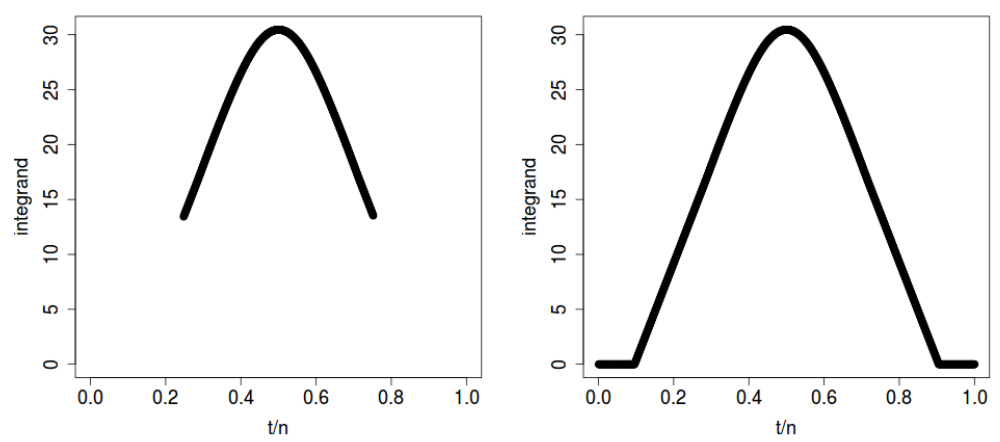

FIG 3. The integrand before (left) and after (right) extrapolation. The integrand can only be directly calculated in the middle part $(t \in[248,752])$, and the outer part is obtained by extending using the boundary tangent. 
the underlying graph being the NNG. We see that the result is quite similar to that based on MST in Tables $1-2$.

TABLE 1

Critical values for the single change-point scan statistic based on NNG at 0.05 significance level. $n=1000$.

\begin{tabular}{|c|c|c|c|c|c|c|c|c|c|c|c|}
\hline & \multicolumn{9}{|c|}{ Critical Values } & \multicolumn{2}{|c|}{ Graph } \\
\hline & \multicolumn{3}{|c|}{$n_{0}=100$} & \multicolumn{3}{|c|}{$n_{0}=50$} & \multicolumn{3}{|c|}{$n_{0}=25$} & \multirow[b]{2}{*}{$\sum\left|G_{i}\right|^{2}$} & \multirow[b]{2}{*}{$d_{\max }$} \\
\hline & A1 & A2 & Per & A1 & A2 & Per & A1 & $\mathrm{A} 2$ & Per & & \\
\hline & 2.96 & 2.98 & 2.95 & 3.04 & 3.07 & 3.03 & 3.10 & 3.13 & 3.08 & 2008 & 2 \\
\hline $\mathrm{N}(0,1)$ & 2.96 & 2.98 & 2.97 & 3.05 & 3.07 & 3.05 & 3.10 & 3.13 & 3.09 & 1972 & 2 \\
\hline \multirow[t]{3}{*}{$d=1$} & 2.96 & 2.98 & 3.01 & 3.04 & 3.07 & 3.10 & 3.10 & 3.12 & 3.13 & 2032 & 2 \\
\hline & 2.96 & 2.98 & 2.97 & 3.04 & 3.07 & 3.04 & 3.10 & 3.13 & 3.09 & 2008 & 2 \\
\hline & 2.96 & 2.99 & 3.01 & 3.05 & 3.08 & 3.10 & 3.10 & 3.13 & 3.13 & 1954 & 2 \\
\hline \multirow{5}{*}{$\begin{array}{c}\operatorname{Exp}(1) \\
d=1\end{array}$} & 2.96 & 2.99 & 2.98 & 3.05 & 3.08 & 3.08 & 3.10 & 3.13 & 3.11 & 1948 & 2 \\
\hline & 2.96 & 2.98 & 2.96 & 3.04 & 3.07 & 3.07 & 3.10 & 3.13 & 3.11 & 2038 & 2 \\
\hline & 2.96 & 2.98 & 2.96 & 3.04 & 3.08 & 3.05 & 3.10 & 3.13 & 3.09 & 2014 & 2 \\
\hline & 2.96 & 2.98 & 2.99 & 3.04 & 3.07 & 3.08 & 3.10 & 3.12 & 3.13 & 2008 & 2 \\
\hline & 2.96 & 2.98 & 2.99 & 3.04 & 3.08 & 3.08 & 3.10 & 3.13 & 3.13 & 2038 & 2 \\
\hline \multirow{5}{*}{$\begin{array}{l}\mathrm{N}(0,1) \\
d=10\end{array}$} & 2.94 & 2.92 & 2.89 & 3.02 & 2.97 & 2.93 & 3.07 & 3.00 & 2.96 & 3370 & 6 \\
\hline & 2.94 & 2.91 & 2.90 & 3.02 & 2.97 & 2.95 & 3.07 & 2.99 & 2.96 & 3502 & 6 \\
\hline & 2.94 & 2.91 & 2.89 & 3.01 & 2.96 & 2.95 & 3.06 & 2.98 & 2.96 & 3444 & 7 \\
\hline & 2.94 & 2.91 & 2.91 & 3.01 & 2.96 & 2.94 & 3.06 & 2.98 & 2.96 & 3436 & 6 \\
\hline & 2.94 & 2.91 & 2.88 & 3.02 & 2.97 & 2.93 & 3.07 & 2.99 & 2.94 & 3330 & 6 \\
\hline \multirow{5}{*}{$\begin{array}{c}\operatorname{Exp}(1) \\
d=10\end{array}$} & 2.94 & 2.92 & 2.91 & 3.02 & 2.98 & 2.96 & 3.07 & 3.00 & 2.98 & 3144 & 5 \\
\hline & 2.94 & 2.92 & 2.92 & 3.02 & 2.98 & 2.97 & 3.07 & 3.00 & 2.99 & 3096 & 6 \\
\hline & 2.94 & 2.92 & 2.92 & 3.02 & 2.98 & 2.98 & 3.07 & 3.01 & 3.01 & 3118 & 6 \\
\hline & 2.94 & 2.93 & 2.92 & 3.02 & 2.98 & 2.97 & 3.07 & 3.01 & 2.99 & 3114 & 5 \\
\hline & 2.94 & 2.92 & 2.91 & 3.02 & 2.98 & 2.98 & 3.07 & 3.01 & 3.00 & 3152 & 6 \\
\hline \multirow{5}{*}{$\begin{array}{l}\mathrm{N}(0,1) \\
d=100\end{array}$} & 2.87 & 2.65 & 2.62 & 2.95 & 2.65 & 2.62 & 3.00 & 2.65 & 2.62 & 9382 & 52 \\
\hline & 2.87 & 2.73 & 2.70 & 2.95 & 2.75 & 2.71 & 3.01 & 2.76 & 2.71 & 8466 & 24 \\
\hline & 2.88 & 2.76 & 2.72 & 2.96 & 2.78 & 2.72 & 3.01 & 2.79 & 2.72 & 7756 & 20 \\
\hline & 2.86 & 2.59 & 2.56 & 2.94 & 2.59 & 2.56 & 3.00 & 2.59 & 2.56 & 11092 & 68 \\
\hline & 2.87 & 2.68 & 2.64 & 2.95 & 2.69 & 2.64 & 3.00 & 2.69 & 2.64 & 9538 & 38 \\
\hline \multirow{5}{*}{$\begin{array}{l}\operatorname{Exp}(1) \\
d=100\end{array}$} & 2.86 & 2.71 & 2.70 & 2.95 & 2.72 & 2.70 & 3.00 & 2.73 & 2.70 & 10222 & 34 \\
\hline & 2.86 & 2.72 & 2.68 & 2.95 & 2.74 & 2.69 & 3.00 & 2.74 & 2.69 & 10390 & 37 \\
\hline & 2.86 & 2.70 & 2.64 & 2.94 & 2.71 & 2.64 & 3.00 & 2.71 & 2.64 & 11574 & 35 \\
\hline & 2.87 & 2.74 & 2.72 & 2.95 & 2.76 & 2.73 & 3.01 & 2.77 & 2.73 & 8782 & 22 \\
\hline & 2.87 & 2.73 & 2.68 & 2.95 & 2.74 & 2.68 & 3.01 & 2.74 & 2.68 & 8622 & 41 \\
\hline
\end{tabular}

C.1.2. A Changed-Interval Alternative. Tables 3 - 7 show the results of $p$-value approximations for the changed interval alternative. The notation and simulation settings are identical to those for the single change-point alternative in Section 3.5, except that $n_{0}$ is replaced by $l_{0}$ for the smallest window size. $\left(l_{1}\right.$ is set to $n-l_{0}$.)

From the tables, conclusions similar to those for the single change-point 
TABLE 2

Critical values for the single change-point scan statistic based on NNG at 0.01 significance level. $n=1000$.

\begin{tabular}{|c|c|c|c|c|c|c|c|c|c|c|c|}
\hline & \multicolumn{9}{|c|}{ Critical Values } & \multicolumn{2}{|c|}{ Graph } \\
\hline & \multicolumn{3}{|c|}{$n_{0}=100$} & \multicolumn{3}{|c|}{$n_{0}=50$} & \multicolumn{3}{|c|}{$n_{0}=25$} & \multirow[b]{2}{*}{$\sum\left|G_{i}\right|^{2}$} & \multirow[b]{2}{*}{$d_{\max }$} \\
\hline & A1 & $\mathrm{A} 2$ & Per & A1 & $\mathrm{A} 2$ & Per & A1 & $\mathrm{A} 2$ & Per & & \\
\hline & 3.50 & 3.53 & 3.53 & 3.57 & 3.61 & 3.59 & 3.61 & 3.65 & 3.63 & 2008 & 2 \\
\hline $\mathrm{N}(0,1)$ & 3.50 & 3.54 & 3.52 & 3.57 & 3.61 & 3.63 & 3.61 & 3.65 & 3.66 & 1972 & 2 \\
\hline \multirow[t]{3}{*}{$d=1$} & 3.50 & 3.53 & 3.58 & 3.57 & 3.61 & 3.66 & 3.61 & 3.65 & 3.71 & 2032 & 2 \\
\hline & 3.50 & 3.53 & 3.56 & 3.57 & 3.61 & 3.63 & 3.61 & 3.65 & 3.68 & 2008 & 2 \\
\hline & 3.50 & 3.54 & 3.53 & 3.57 & 3.62 & 3.64 & 3.61 & 3.66 & 3.65 & 1954 & 2 \\
\hline \multirow{5}{*}{$\begin{array}{c}\operatorname{Exp}(1) \\
d=1\end{array}$} & 3.50 & 3.54 & 3.50 & 3.57 & 3.62 & 3.61 & 3.61 & 3.66 & 3.64 & 1948 & 2 \\
\hline & 3.50 & 3.53 & 3.57 & 3.57 & 3.61 & 3.63 & 3.61 & 3.65 & 3.65 & 2038 & 2 \\
\hline & 3.50 & 3.54 & 3.52 & 3.57 & 3.61 & 3.63 & 3.61 & 3.66 & 3.66 & 2014 & 2 \\
\hline & 3.50 & 3.53 & 3.60 & 3.57 & 3.61 & 3.66 & 3.61 & 3.65 & 3.71 & 2008 & 2 \\
\hline & 3.50 & 3.54 & 3.54 & 3.57 & 3.62 & 3.58 & 3.61 & 3.66 & 3.66 & 2038 & 2 \\
\hline \multirow{5}{*}{$\begin{array}{l}\mathrm{N}(0,1) \\
d=10\end{array}$} & 3.48 & 3.45 & 3.46 & 3.55 & 3.49 & 3.48 & 3.59 & 3.50 & 3.49 & 3370 & 6 \\
\hline & 3.48 & 3.44 & 3.47 & 3.54 & 3.48 & 3.48 & 3.59 & 3.49 & 3.48 & 3502 & 6 \\
\hline & 3.48 & 3.44 & 3.42 & 3.54 & 3.47 & 3.45 & 3.58 & 3.48 & 3.46 & 3444 & 7 \\
\hline & 3.48 & 3.44 & 3.43 & 3.54 & 3.47 & 3.46 & 3.59 & 3.48 & 3.47 & 3436 & 6 \\
\hline & 3.48 & 3.44 & 3.44 & 3.55 & 3.48 & 3.48 & 3.59 & 3.49 & 3.48 & 3330 & 6 \\
\hline \multirow{5}{*}{$\begin{array}{l}\operatorname{Exp}(1) \\
d=10\end{array}$} & 3.49 & 3.45 & 3.46 & 3.55 & 3.49 & 3.51 & 3.59 & 3.50 & 3.51 & 3144 & 5 \\
\hline & 3.49 & 3.45 & 3.48 & 3.55 & 3.49 & 3.52 & 3.59 & 3.50 & 3.52 & 3096 & 6 \\
\hline & 3.49 & 3.46 & 3.48 & 3.55 & 3.49 & 3.54 & 3.59 & 3.51 & 3.57 & 3118 & 6 \\
\hline & 3.49 & 3.46 & 3.41 & 3.55 & 3.50 & 3.46 & 3.59 & 3.51 & 3.46 & 3114 & 5 \\
\hline & 3.49 & 3.46 & 3.49 & 3.55 & 3.49 & 3.52 & 3.59 & 3.51 & 3.53 & 3152 & 6 \\
\hline \multirow{5}{*}{$\begin{array}{c}\mathrm{N}(0,1) \\
d=100\end{array}$} & 3.42 & 3.13 & 3.07 & 3.49 & 3.13 & 3.07 & 3.54 & 3.13 & 3.07 & 9382 & 52 \\
\hline & 3.43 & 3.21 & 3.19 & 3.50 & 3.21 & 3.19 & 3.54 & 3.21 & 3.19 & 8466 & 24 \\
\hline & 3.44 & 3.25 & 3.23 & 3.50 & 3.25 & 3.23 & 3.54 & 3.25 & 3.23 & 7756 & 20 \\
\hline & 3.42 & 3.09 & 3.08 & 3.48 & 3.09 & 3.08 & 3.53 & 3.09 & 3.08 & 11092 & 68 \\
\hline & 3.42 & 3.16 & 3.16 & 3.49 & 3.16 & 3.16 & 3.54 & 3.16 & 3.16 & 9538 & 38 \\
\hline \multirow{5}{*}{$\begin{array}{c}\operatorname{Exp}(1) \\
d=100\end{array}$} & 3.42 & 3.20 & 3.19 & 3.49 & 3.20 & 3.19 & 3.53 & 3.20 & 3.19 & 10222 & 34 \\
\hline & 3.42 & 3.22 & 3.21 & 3.49 & 3.22 & 3.21 & 3.53 & 3.22 & 3.21 & 10390 & 37 \\
\hline & 3.42 & 3.18 & 3.17 & 3.48 & 3.18 & 3.17 & 3.53 & 3.18 & 3.17 & 11574 & 35 \\
\hline & 3.43 & 3.23 & 3.23 & 3.49 & 3.23 & 3.23 & 3.54 & 3.23 & 3.23 & 8782 & 22 \\
\hline & 3.43 & 3.22 & 3.24 & 3.50 & 3.22 & 3.24 & 3.54 & 3.22 & 3.24 & 8622 & 41 \\
\hline
\end{tabular}


alternative can be drawn. The analytic approximation after skewness correction performs much better than the analytic approximation under Gaussian assumption, especially when dimension increases. The accuracy of skewcorrected approximation does not degrade significantly with dimension. It does well for MST- and NNG- based tests when the smallest window size to be considered is as small as 25 for both 0.05 and 0.01 significance levels, and for MDP-based test when the smallest window size is 50 .

TABLE 3

Critical values for the changed interval scan statistic based on MST at 0.05 significance level. $n=1000$.

\begin{tabular}{|c|c|c|c|c|c|c|c|c|c|c|c|}
\hline & \multicolumn{9}{|c|}{ Critical Values } & \multicolumn{2}{|c|}{ Graph } \\
\hline & \multicolumn{3}{|c|}{$l_{0}=100$} & \multicolumn{3}{|c|}{$l_{0}=50$} & \multicolumn{3}{|c|}{$l_{0}=25$} & \multirow[b]{2}{*}{$\sum\left|G_{i}\right|^{2}$} & \multirow[b]{2}{*}{$d_{\max }$} \\
\hline & A1 & $\mathrm{A} 2$ & Per & A1 & $\mathrm{A} 2$ & Per & A1 & $\mathrm{A} 2$ & Per & & \\
\hline$d=1$ & 4.08 & 4.29 & 4.24 & 4.22 & 4.76 & 4.73 & 4.33 & 5.44 & 5.77 & 4994 & 2 \\
\hline \multirow{5}{*}{$\begin{array}{l}\mathrm{N}(0,1) \\
d=10\end{array}$} & 3.97 & 3.89 & 3.84 & 4.07 & 3.92 & 3.89 & 4.16 & 3.93 & 3.89 & 5454 & 8 \\
\hline & 3.97 & 3.91 & 3.81 & 4.07 & 3.95 & 3.85 & 4.16 & 3.97 & 3.87 & 5400 & 7 \\
\hline & 3.97 & 3.90 & 3.81 & 4.07 & 3.93 & 3.90 & 4.16 & 3.94 & 3.91 & 5448 & 8 \\
\hline & 3.97 & 3.90 & 3.91 & 4.07 & 3.94 & 3.93 & 4.16 & 3.95 & 3.94 & 5440 & 7 \\
\hline & 3.97 & 3.89 & 3.82 & 4.07 & 3.91 & 3.85 & 4.15 & 3.93 & 3.85 & 5524 & 8 \\
\hline \multirow{5}{*}{$\begin{array}{l}\operatorname{Exp}(1) \\
d=10\end{array}$} & 3.99 & 3.93 & 3.86 & 4.09 & 3.97 & 3.92 & 4.17 & 3.99 & 3.95 & 5042 & 8 \\
\hline & 3.99 & 3.93 & 3.84 & 4.09 & 3.96 & 3.90 & 4.17 & 4.00 & 3.92 & 5040 & 6 \\
\hline & 3.99 & 3.93 & 3.85 & 4.09 & 3.97 & 3.91 & 4.17 & 4.00 & 3.93 & 5106 & 6 \\
\hline & 3.99 & 3.93 & 3.82 & 4.09 & 3.97 & 3.87 & 4.17 & 3.99 & 3.91 & 5042 & 6 \\
\hline & 3.99 & 3.91 & 3.94 & 4.08 & 3.95 & 3.98 & 4.17 & 3.97 & 3.98 & 5126 & 8 \\
\hline \multirow{5}{*}{$\begin{array}{c}\mathrm{N}(0,1) \\
d=100\end{array}$} & 3.87 & 3.51 & 3.52 & 3.98 & 3.51 & 3.52 & 4.09 & 3.51 & 3.52 & 11600 & 40 \\
\hline & 3.86 & 3.49 & 3.55 & 3.98 & 3.49 & 3.55 & 4.08 & 3.49 & 3.55 & 13346 & 64 \\
\hline & 3.88 & 3.57 & 3.66 & 3.99 & 3.57 & 3.66 & 4.09 & 3.57 & 3.66 & 10422 & 34 \\
\hline & 3.88 & 3.57 & 3.58 & 3.99 & 3.57 & 3.58 & 4.09 & 3.57 & 3.58 & 10804 & 43 \\
\hline & 3.88 & 3.56 & 3.58 & 3.99 & 3.56 & 3.58 & 4.09 & 3.56 & 3.58 & 10862 & 36 \\
\hline \multirow{5}{*}{$\begin{array}{l}\operatorname{Exp}(1) \\
d=100\end{array}$} & 3.88 & 3.63 & 3.59 & 3.99 & 3.63 & 3.59 & 4.09 & 3.63 & 3.59 & 10384 & 24 \\
\hline & 3.87 & 3.58 & 3.49 & 3.98 & 3.58 & 3.49 & 4.09 & 3.58 & 3.49 & 11922 & 33 \\
\hline & 3.88 & 3.60 & 3.63 & 3.99 & 3.60 & 3.63 & 4.09 & 3.60 & 3.63 & 11194 & 34 \\
\hline & 3.89 & 3.63 & 3.55 & 4.00 & 3.63 & 3.55 & 4.10 & 3.63 & 3.55 & 9680 & 27 \\
\hline & 3.88 & 3.62 & 3.60 & 3.99 & 3.62 & 3.60 & 4.09 & 3.62 & 3.60 & 10468 & 29 \\
\hline
\end{tabular}

C.2. Coverage Probability. Here, we show extra boxplots of coverage probability for the single change-point alternative. We see that the skewness correction helps in general. 
TABLE 4

Critical values for the changed interval scan statistic based on MST at 0.01 significance level. $n=1000$.

\begin{tabular}{|c|c|c|c|c|c|c|c|c|c|c|c|}
\hline & \multicolumn{9}{|c|}{ Critical Values } & \multicolumn{2}{|c|}{ Graph } \\
\hline & \multicolumn{3}{|c|}{$l_{0}=100$} & \multicolumn{3}{|c|}{$l_{0}=50$} & \multicolumn{3}{|c|}{$l_{0}=25$} & \multirow[b]{2}{*}{$\sum\left|G_{i}\right|^{2}$} & \multirow[b]{2}{*}{$d_{\max }$} \\
\hline & A1 & A2 & Per & A1 & $\mathrm{A} 2$ & Per & A1 & A2 & Per & & \\
\hline$d=1$ & 4.51 & 4.78 & 4.73 & 4.63 & 5.31 & 5.30 & 4.72 & 6.08 & 6.65 & 4994 & 2 \\
\hline \multirow{5}{*}{$\begin{array}{l}\mathrm{N}(0,1) \\
d=10\end{array}$} & 4.42 & 4.32 & 4.31 & 4.50 & 4.33 & 4.33 & 4.58 & 4.33 & 4.33 & 5454 & 8 \\
\hline & 4.42 & 4.34 & 4.22 & 4.51 & 4.36 & 4.25 & 4.58 & 4.37 & 4.25 & 5400 & 7 \\
\hline & 4.42 & 4.33 & 4.20 & 4.50 & 4.51 & 4.25 & 4.58 & 4.35 & 4.29 & 5448 & 8 \\
\hline & 4.42 & 4.34 & 4.36 & 4.50 & 4.32 & 4.36 & 4.58 & 4.36 & 4.36 & 5440 & 7 \\
\hline & 4.42 & 4.32 & 4.31 & 4.50 & 4.33 & 4.32 & 4.57 & 4.33 & 4.32 & 5524 & 8 \\
\hline \multirow{5}{*}{$\begin{array}{l}\operatorname{Exp}(1) \\
d=10\end{array}$} & 4.43 & 4.36 & 4.36 & 4.52 & 4.39 & 4.36 & 4.59 & 4.39 & 4.36 & 5042 & 8 \\
\hline & 4.43 & 4.36 & 4.30 & 4.52 & 4.39 & 4.36 & 4.59 & 4.40 & 4.36 & 5040 & 6 \\
\hline & 4.43 & 4.36 & 4.32 & 4.52 & 4.39 & 4.38 & 4.59 & 4.40 & 4.44 & 5106 & 6 \\
\hline & 4.43 & 4.36 & 4.27 & 4.52 & 4.39 & 4.33 & 4.59 & 4.39 & 4.33 & 5042 & 6 \\
\hline & 4.43 & 4.35 & 4.35 & 4.52 & 4.37 & 4.35 & 4.59 & 4.37 & 4.35 & 5126 & 8 \\
\hline \multirow{5}{*}{$\begin{array}{c}\mathrm{N}(0,1) \\
d=100\end{array}$} & 4.34 & 3.99 & 4.28 & 4.43 & 3.99 & 4.28 & 4.52 & 3.99 & 4.28 & 11600 & 40 \\
\hline & 4.33 & 3.98 & 3.95 & 4.42 & 3.98 & 3.95 & 4.51 & 3.98 & 3.95 & 13346 & 64 \\
\hline & 4.34 & 4.04 & 4.12 & 4.44 & 4.04 & 4.12 & 4.52 & 4.04 & 4.12 & 10422 & 34 \\
\hline & 4.34 & 4.05 & 4.22 & 4.43 & 4.05 & 4.22 & 4.52 & 4.05 & 4.22 & 10804 & 43 \\
\hline & 4.34 & 4.03 & 4.00 & 4.43 & 4.03 & 4.00 & 4.52 & 4.03 & 4.00 & 10862 & 36 \\
\hline \multirow{5}{*}{$\begin{array}{l}\operatorname{Exp}(1) \\
d=100\end{array}$} & 4.34 & 4.10 & 3.95 & 4.44 & 4.10 & 3.95 & 4.52 & 4.10 & 3.95 & 10384 & 24 \\
\hline & 4.33 & 4.05 & 3.87 & 4.43 & 4.05 & 3.87 & 4.52 & 4.05 & 3.87 & 11922 & 33 \\
\hline & 4.34 & 4.08 & 4.14 & 4.43 & 4.08 & 4.14 & 4.52 & 4.08 & 4.14 & 11194 & 34 \\
\hline & 4.35 & 4.10 & 3.86 & 4.44 & 4.10 & 3.86 & 4.53 & 4.10 & 3.86 & 9680 & 27 \\
\hline & 4.34 & 4.08 & 4.10 & 4.44 & 4.08 & 4.10 & 4.52 & 4.08 & 4.10 & 10468 & 29 \\
\hline
\end{tabular}

TABLE 5

Critical values for the changed interval scan statistic based on MDP. $n=1000$.

\begin{tabular}{c|cc|cc|cc|cc}
\multicolumn{8}{c|}{ significance level $=0.05$} \\
\hline \hline & \multicolumn{2}{|c|}{$d=1$} & \multicolumn{2}{c|}{$d=10$} & \multicolumn{2}{c}{$d=100$} \\
\hline$l_{0}$ & A1 & A2 & $\mathrm{N}(0,1)$ & $\operatorname{Exp}(1)$ & $\mathrm{N}(0,1)$ & $\operatorname{Exp}(1)$ & $\mathrm{N}(0,1)$ & $\operatorname{Exp}(1)$ \\
\hline \hline 100 & 4.08 & 4.38 & 4.39 & 4.46 & 4.30 & 4.29 & 4.32 & 4.32 \\
\hline 50 & 4.22 & 4.97 & 5.03 & 5.12 & 5.10 & 4.87 & 5.19 & 4.99 \\
\hline 25 & 4.33 & 5.81 & 6.31 & 6.32 & 6.14 & 6.12 & 6.60 & 6.35 \\
\hline \hline
\end{tabular}

significance level $=0.01$

\begin{tabular}{c|cc|cc|cc|cc}
\hline \hline & & & \multicolumn{2}{|c|}{$d=1$} & \multicolumn{2}{c|}{$d=10$} & \multicolumn{2}{c}{$d=100$} \\
\hline$l_{0}$ & $\mathrm{~A} 1$ & $\mathrm{~A} 2$ & $\mathrm{~N}(0,1)$ & $\operatorname{Exp}(1)$ & $\mathrm{N}(0,1)$ & $\operatorname{Exp}(1)$ & $\mathrm{N}(0,1)$ & $\operatorname{Exp}(1)$ \\
\hline \hline 100 & 4.51 & 4.90 & 4.91 & 5.13 & 4.93 & 4.92 & 5.01 & 4.91 \\
\hline 50 & 4.63 & 5.58 & 5.63 & 5.94 & 5.64 & 5.48 & 6.13 & 5.63 \\
\hline 25 & 4.72 & 6.52 & 6.91 & 6.91 & 6.91 & 6.91 & 7.12 & 6.91 \\
\hline \hline
\end{tabular}


TABle 6

Critical values for the changed interval scan statistic based on NNG at 0.05 significance level. $n=1000$.

\begin{tabular}{|c|c|c|c|c|c|c|c|c|c|c|c|}
\hline & \multicolumn{9}{|c|}{ Critical Values } & \multicolumn{2}{|c|}{ Graph } \\
\hline & \multicolumn{3}{|c|}{$l_{0}=100$} & \multicolumn{3}{|c|}{$l_{0}=50$} & \multicolumn{3}{|c|}{$l_{0}=25$} & \multirow[b]{2}{*}{$\sum\left|G_{i}\right|^{2}$} & \multirow[b]{2}{*}{$d_{\max }$} \\
\hline & A1 & $\mathrm{A} 2$ & Per & A1 & $\mathrm{A} 2$ & Per & A1 & $\mathrm{A} 2$ & Per & & \\
\hline & 4.04 & 4.10 & 4.07 & 4.15 & 4.23 & 4.20 & 4.23 & 4.31 & 4.30 & 2026 & 2 \\
\hline $\mathrm{N}(0,1)$ & 4.04 & 4.10 & 4.09 & 4.15 & 4.24 & 4.18 & 4.23 & 4.31 & 4.24 & 1942 & 2 \\
\hline \multirow[t]{3}{*}{$d=1$} & 4.04 & 4.10 & 4.11 & 4.15 & 4.24 & 4.23 & 4.23 & 4.31 & 4.35 & 1948 & 2 \\
\hline & 4.04 & 4.10 & 3.96 & 4.15 & 4.23 & 4.11 & 4.23 & 4.31 & 4.25 & 2038 & 2 \\
\hline & 4.04 & 4.10 & 4.04 & 4.15 & 4.24 & 4.17 & 4.23 & 4.31 & 4.31 & 1960 & 2 \\
\hline & 4.04 & 4.10 & 4.00 & 4.15 & 4.23 & 4.14 & 4.23 & 4.31 & 4.24 & 2086 & 2 \\
\hline $\operatorname{Exp}(1)$ & 4.04 & 4.10 & 4.08 & 4.15 & 4.23 & 4.20 & 4.23 & 4.31 & 4.24 & 1990 & 2 \\
\hline \multirow[t]{3}{*}{$d=1$} & 4.04 & 4.10 & 4.00 & 4.15 & 4.24 & 4.15 & 4.23 & 4.32 & 4.27 & 2014 & 2 \\
\hline & 4.04 & 4.10 & 4.01 & 4.15 & 4.23 & 4.20 & 4.23 & 4.31 & 4.34 & 2080 & 2 \\
\hline & 4.04 & 4.10 & 4.04 & 4.15 & 4.23 & 4.18 & 4.23 & 4.31 & 4.27 & 2008 & 2 \\
\hline & 3.99 & 3.92 & 3.82 & 4.09 & 3.96 & 3.88 & 4.18 & 3.97 & 3.90 & 3558 & 6 \\
\hline $\mathrm{N}(0,1)$ & 3.99 & 3.91 & 3.86 & 4.09 & 3.94 & 3.86 & 4.18 & 3.95 & 3.88 & 3508 & 6 \\
\hline \multirow[t]{3}{*}{$d=10$} & 4.00 & 3.92 & 3.86 & 4.10 & 3.96 & 3.93 & 4.18 & 3.97 & 3.93 & 3394 & 6 \\
\hline & 3.99 & 3.91 & 3.81 & 4.09 & 3.94 & 3.86 & 4.18 & 3.95 & 3.90 & 3418 & 6 \\
\hline & 3.99 & 3.91 & 3.88 & 4.09 & 3.94 & 3.88 & 4.18 & 3.95 & 3.88 & 3450 & 6 \\
\hline & 4.00 & 3.94 & 3.85 & 4.10 & 3.98 & 3.91 & 4.18 & 3.99 & 3.91 & 3306 & 6 \\
\hline $\operatorname{Exp}(1)$ & 4.01 & 3.95 & 3.91 & 4.11 & 4.00 & 3.98 & 4.19 & 4.02 & 3.99 & 3118 & 5 \\
\hline \multirow{3}{*}{$d=10$} & 4.00 & 3.94 & 3.89 & 4.10 & 3.98 & 3.93 & 4.19 & 4.00 & 3.94 & 3018 & 5 \\
\hline & 4.00 & 3.95 & 3.90 & 4.11 & 3.99 & 3.93 & 4.19 & 4.01 & 3.93 & 3014 & 5 \\
\hline & 4.01 & 3.96 & 3.95 & 4.11 & 4.01 & 3.97 & 4.19 & 4.03 & 3.99 & 3092 & 5 \\
\hline \multirow{5}{*}{$\begin{array}{c}\mathrm{N}(0,1) \\
d=100\end{array}$} & 3.89 & 3.55 & 3.48 & 4.00 & 3.55 & 3.48 & 4.10 & 3.55 & 3.48 & 8240 & 30 \\
\hline & 3.88 & 3.50 & 3.49 & 3.99 & 3.50 & 3.49 & 4.09 & 3.50 & 3.49 & 9360 & 33 \\
\hline & 3.90 & 3.61 & 3.60 & 4.00 & 3.61 & 3.60 & 4.10 & 3.61 & 3.60 & 8482 & 18 \\
\hline & 3.88 & 3.51 & 3.48 & 3.99 & 3.51 & 3.48 & 4.09 & 3.51 & 3.48 & 9154 & 40 \\
\hline & 3.88 & 3.50 & 3.44 & 3.99 & 3.50 & 3.44 & 4.09 & 3.50 & 3.44 & 9392 & 39 \\
\hline \multirow{5}{*}{$\begin{array}{l}\operatorname{Exp}(1) \\
d=100\end{array}$} & 3.88 & 3.54 & 3.47 & 3.99 & 3.54 & 3.47 & 4.09 & 3.54 & 3.47 & 10406 & 45 \\
\hline & 3.88 & 3.55 & 3.55 & 3.99 & 3.55 & 3.55 & 4.09 & 3.55 & 3.55 & 10504 & 44 \\
\hline & 3.88 & 3.54 & 3.61 & 3.99 & 3.54 & 3.61 & 4.09 & 3.54 & 3.61 & 10106 & 32 \\
\hline & 3.90 & 3.64 & 3.53 & 4.00 & 3.63 & 3.53 & 4.10 & 3.63 & 3.53 & 8666 & 22 \\
\hline & 3.90 & 3.58 & 3.57 & 4.00 & 3.58 & 3.57 & 4.10 & 3.58 & 3.57 & 8274 & 28 \\
\hline
\end{tabular}


TABLE 7

Critical values for the changed interval scan statistic based on NNG at 0.01 significance level. $n=1000$.

\begin{tabular}{|c|c|c|c|c|c|c|c|c|c|c|c|}
\hline & \multicolumn{9}{|c|}{ Critical Values } & \multicolumn{2}{|c|}{ Graph } \\
\hline & \multicolumn{3}{|c|}{$l_{0}=100$} & \multicolumn{3}{|c|}{$l_{0}=50$} & \multicolumn{3}{|c|}{$l_{0}=25$} & \multirow[b]{2}{*}{$\sum\left|G_{i}\right|^{2}$} & \multirow[b]{2}{*}{$d_{\max }$} \\
\hline & A1 & $\mathrm{A} 2$ & Per & A1 & $\mathrm{A} 2$ & Per & A1 & $\mathrm{A} 2$ & Per & & \\
\hline & 4.48 & 4.55 & 4.58 & 4.57 & 4.67 & 4.65 & 4.64 & 4.73 & 4.65 & 2026 & 2 \\
\hline $\mathrm{N}(0,1)$ & 4.48 & 4.56 & 4.53 & 4.57 & 4.68 & 4.71 & 4.64 & 4.74 & 4.79 & 1942 & 2 \\
\hline \multirow[t]{3}{*}{$d=1$} & 4.48 & 4.56 & 4.56 & 4.57 & 4.68 & 4.72 & 4.64 & 4.74 & 4.83 & 1948 & 2 \\
\hline & 4.48 & 4.55 & 4.45 & 4.57 & 4.67 & 4.68 & 4.64 & 4.74 & 4.69 & 2038 & 2 \\
\hline & 4.48 & 4.56 & 4.56 & 4.57 & 4.68 & 4.66 & 4.64 & 4.74 & 4.82 & 1960 & 2 \\
\hline & 4.48 & 4.55 & 4.49 & 4.57 & 4.67 & 4.62 & 4.64 & 4.74 & 4.68 & 2086 & 2 \\
\hline $\operatorname{Exp}(1)$ & 4.48 & 4.55 & 4.49 & 4.57 & 4.67 & 4.57 & 4.64 & 4.73 & 4.57 & 1990 & 2 \\
\hline \multirow[t]{3}{*}{$d=1$} & 4.48 & 4.56 & 4.49 & 4.57 & 4.68 & 4.59 & 4.64 & 4.75 & 4.60 & 2014 & 2 \\
\hline & 4.48 & 4.55 & 4.61 & 4.57 & 4.67 & 4.65 & 4.64 & 4.74 & 4.76 & 2080 & 2 \\
\hline & 4.48 & 4.55 & 4.60 & 4.57 & 4.67 & 4.65 & 4.64 & 4.73 & 4.78 & 2008 & 2 \\
\hline & 4.44 & 4.35 & 4.20 & 4.52 & 4.39 & 4.25 & 4.60 & 4.37 & 4.25 & 3558 & 6 \\
\hline $\mathrm{N}(0,1)$ & 4.44 & 4.34 & 4.34 & 4.52 & 4.35 & 4.38 & 4.59 & 4.35 & 4.38 & 3508 & 6 \\
\hline \multirow[t]{3}{*}{$d=10$} & 4.44 & 4.35 & 4.28 & 4.52 & 4.36 & 4.33 & 4.60 & 4.37 & 4.33 & 3394 & 6 \\
\hline & 4.44 & 4.34 & 4.30 & 4.52 & 4.36 & 4.30 & 4.59 & 4.35 & 4.30 & 3418 & 6 \\
\hline & 4.44 & 4.34 & 4.22 & 4.52 & 4.35 & 4.22 & 4.59 & 4.35 & 4.22 & 3450 & 6 \\
\hline & 4.44 & 4.37 & 4.31 & 4.53 & 4.43 & 4.39 & 4.60 & 4.39 & 4.39 & 3306 & 6 \\
\hline $\operatorname{Exp}(1)$ & 4.45 & 4.38 & 4.39 & 4.53 & 4.42 & 4.50 & 4.60 & 4.42 & 4.50 & 3118 & 5 \\
\hline \multirow[t]{3}{*}{$d=10$} & 4.45 & 4.37 & 4.31 & 4.53 & 4.72 & 4.33 & 4.60 & 4.39 & 4.38 & 3018 & 5 \\
\hline & 4.45 & 4.38 & 4.42 & 4.53 & 4.35 & 4.45 & 4.60 & 4.41 & 4.45 & 3014 & 5 \\
\hline & 4.45 & 4.39 & 4.46 & 4.53 & 4.43 & 4.47 & 4.61 & 4.43 & 4.47 & 3092 & 5 \\
\hline \multirow{5}{*}{$\begin{array}{c}\mathrm{N}(0,1) \\
d=100\end{array}$} & 4.35 & 4.02 & 3.91 & 4.44 & 4.02 & 3.91 & 4.53 & 4.02 & 3.91 & 8240 & 30 \\
\hline & 4.34 & 3.97 & 3.82 & 4.44 & 3.97 & 3.82 & 4.52 & 3.97 & 3.82 & 9360 & 33 \\
\hline & 4.36 & 4.07 & 3.94 & 4.45 & 4.07 & 3.94 & 4.53 & 4.07 & 3.94 & 8482 & 18 \\
\hline & 4.35 & 3.99 & 4.06 & 4.44 & 3.99 & 4.06 & 4.52 & 3.99 & 4.06 & 9154 & 40 \\
\hline & 4.34 & 3.98 & 3.83 & 4.44 & 3.98 & 3.83 & 4.52 & 3.98 & 3.83 & 9392 & 39 \\
\hline \multirow{5}{*}{$\begin{array}{l}\operatorname{Exp}(1) \\
d=100\end{array}$} & 4.34 & 4.02 & 3.87 & 4.43 & 4.02 & 3.87 & 4.52 & 4.02 & 3.87 & 10406 & 45 \\
\hline & 4.34 & 4.03 & 3.99 & 4.43 & 4.03 & 3.99 & 4.52 & 4.03 & 3.99 & 10504 & 44 \\
\hline & 4.34 & 4.02 & 4.22 & 4.43 & 4.02 & 4.22 & 4.52 & 4.02 & 4.22 & 10106 & 32 \\
\hline & 4.35 & 4.10 & 3.95 & 4.44 & 4.10 & 3.95 & 4.53 & 4.10 & 3.95 & 8666 & 22 \\
\hline & 4.36 & 4.05 & 4.02 & 4.45 & 4.05 & 4.02 & 4.53 & 4.05 & 4.02 & 8274 & 28 \\
\hline
\end{tabular}



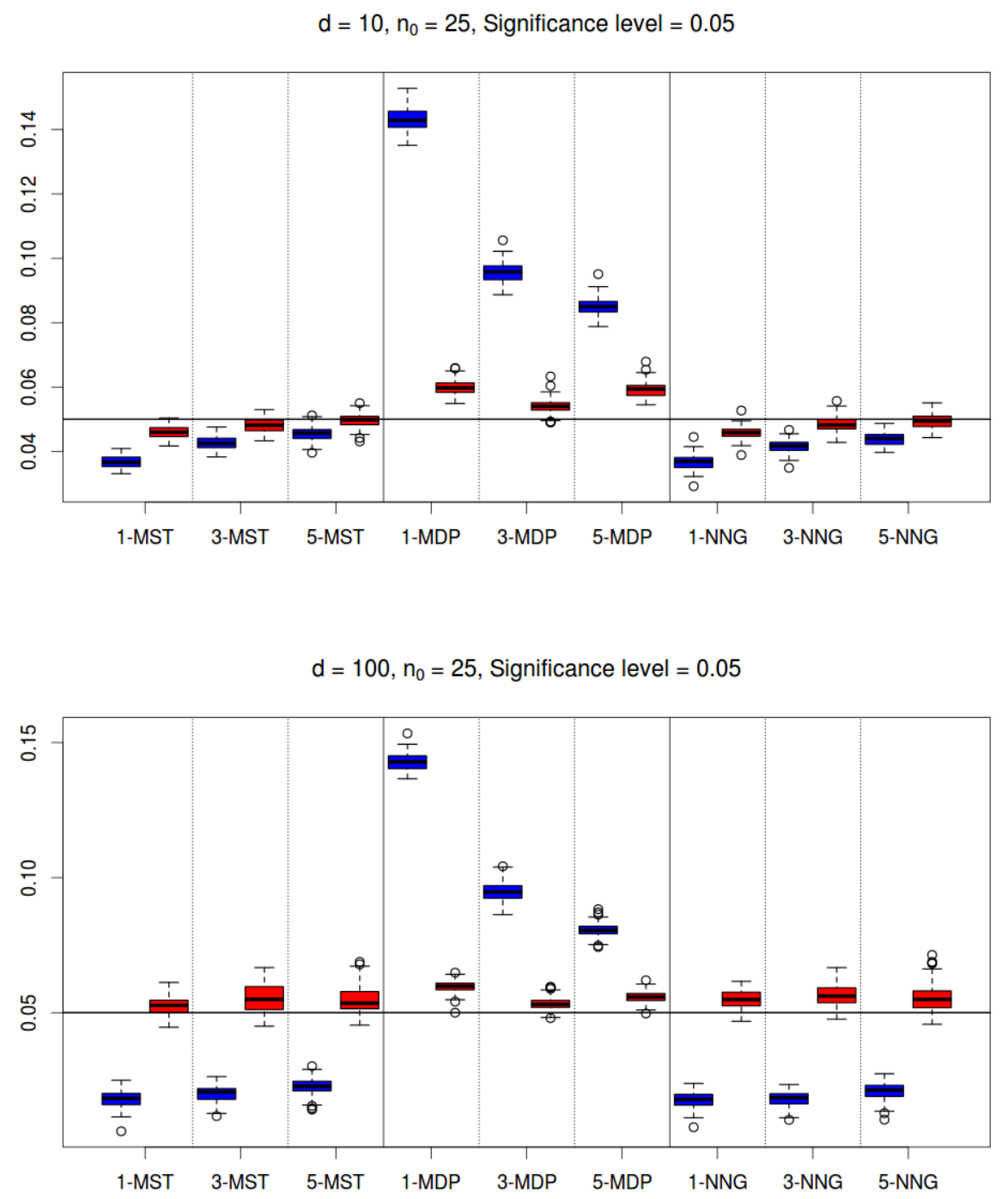

FIG 4. The counterpart boxplots of Figure 7 with the smallest window size being 25 and significance level 0.05 . 

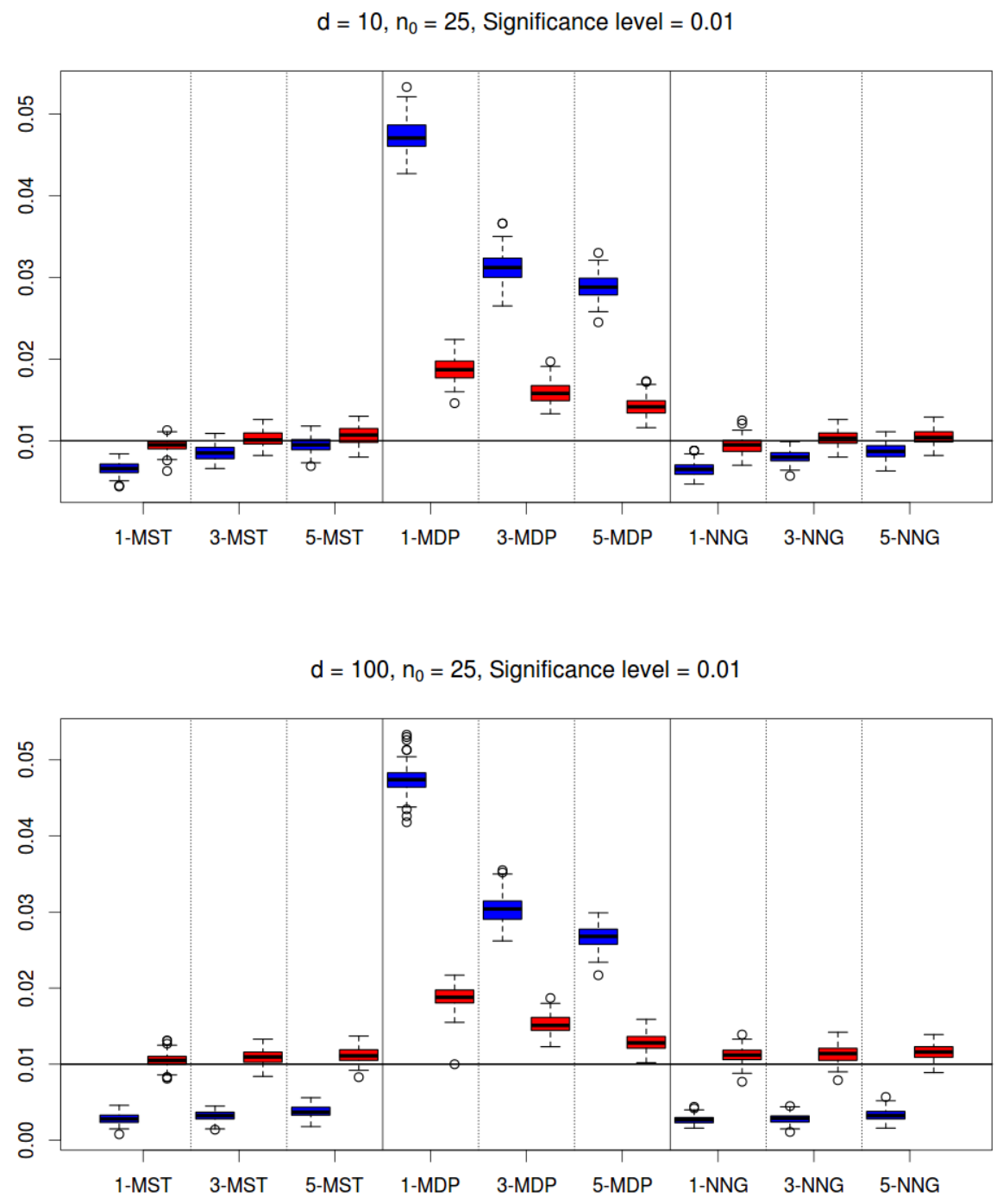

FIG 5. The counterpart boxplots of Figure 7 with the smallest window size being 25 and significance level 0.01 . 

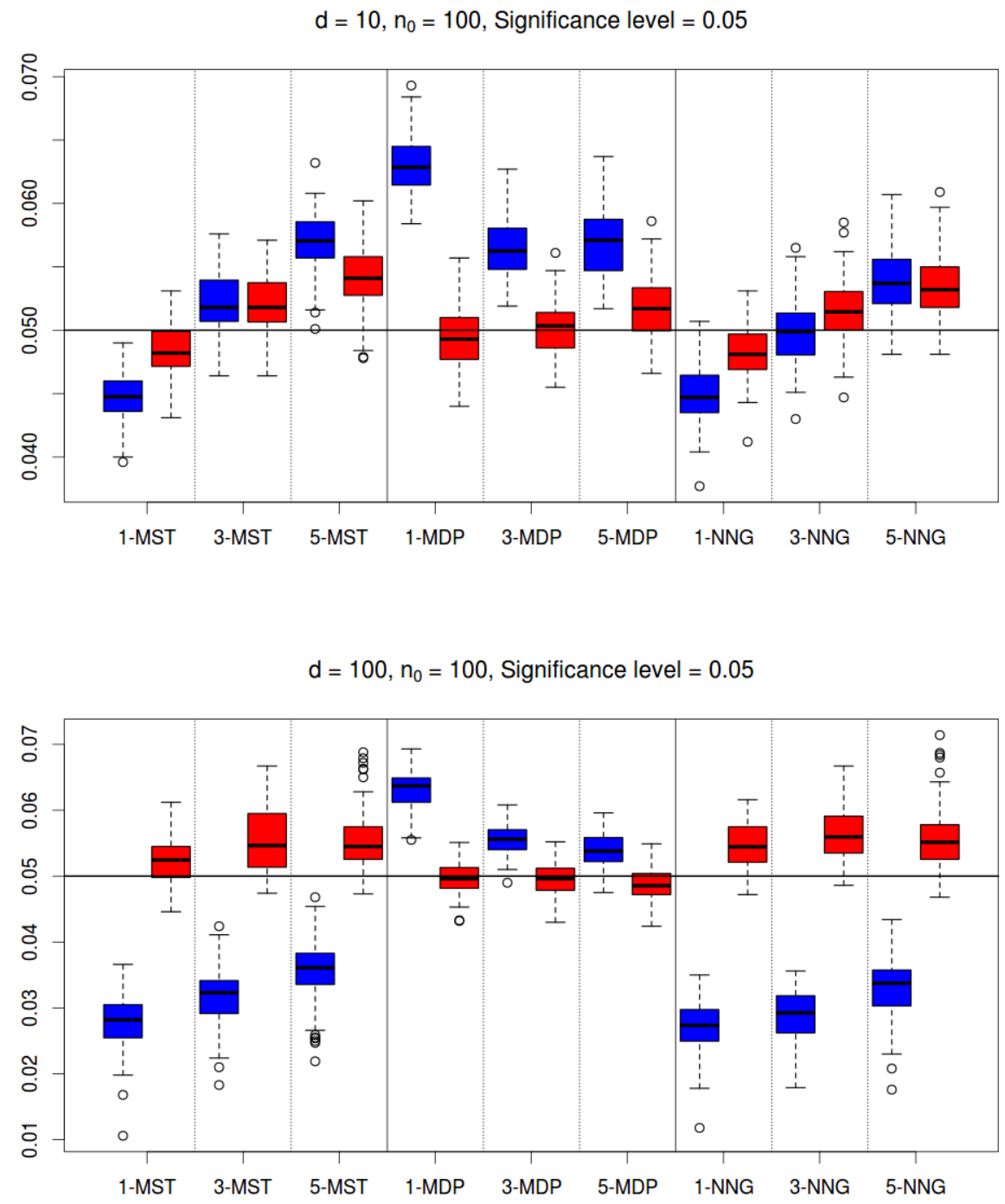

FIG 6. The counterpart boxplots of Figure 7 with the smallest window size being 100 and significance level 0.05 . 

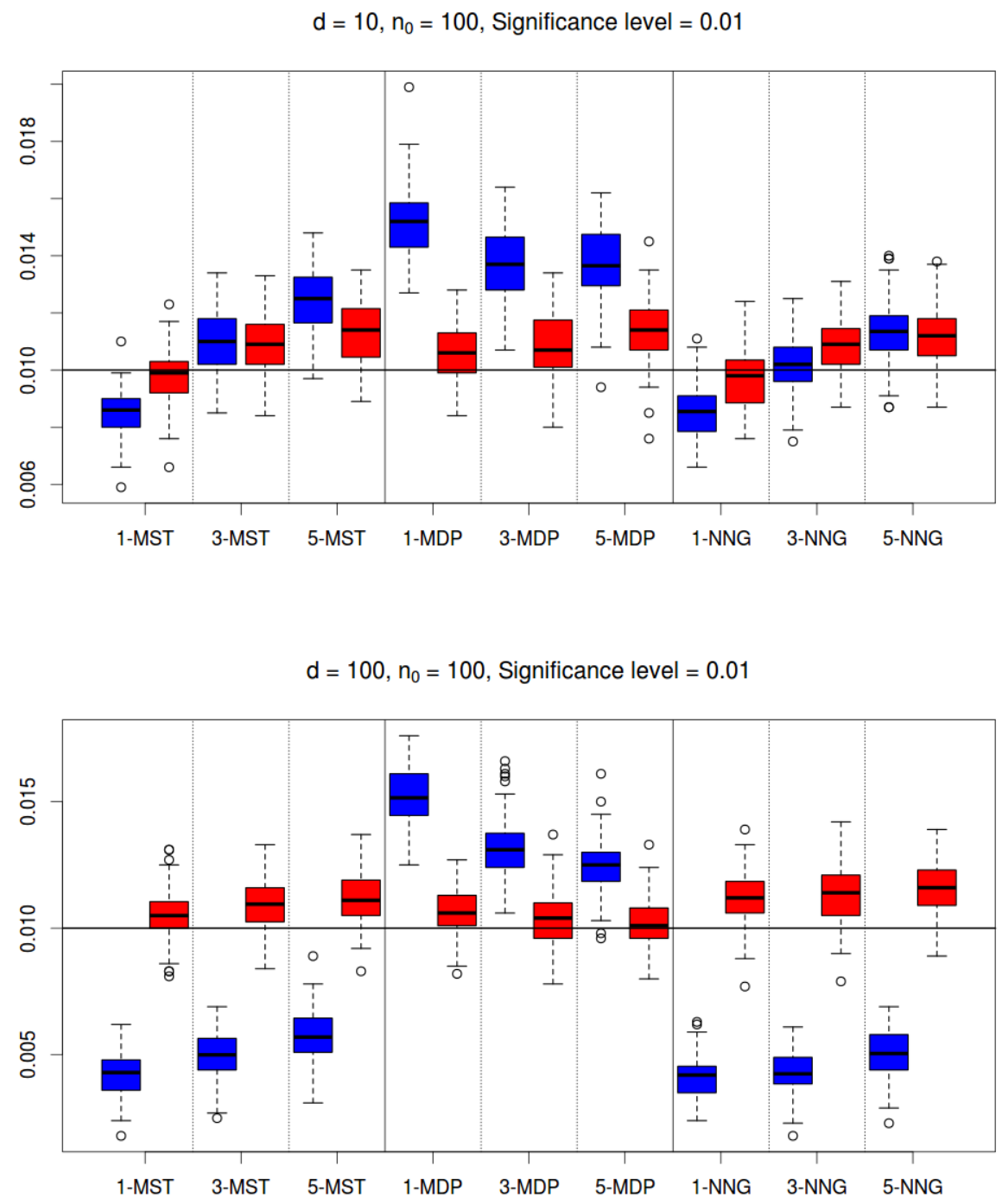

FIG 7. The counterpart boxplots of Figure 7 with the smallest window size being 100 and significance level 0.01 . 


\section{SUPPLEMENT D: BLOCK PERMUTATION RESULTS}

\section{D.1. Authorship Data.}

D.1.1. Scan over the Entire Book. Here, we show plots for $Z_{G, b p}$ for the authorship data under block size 2 and 10 .
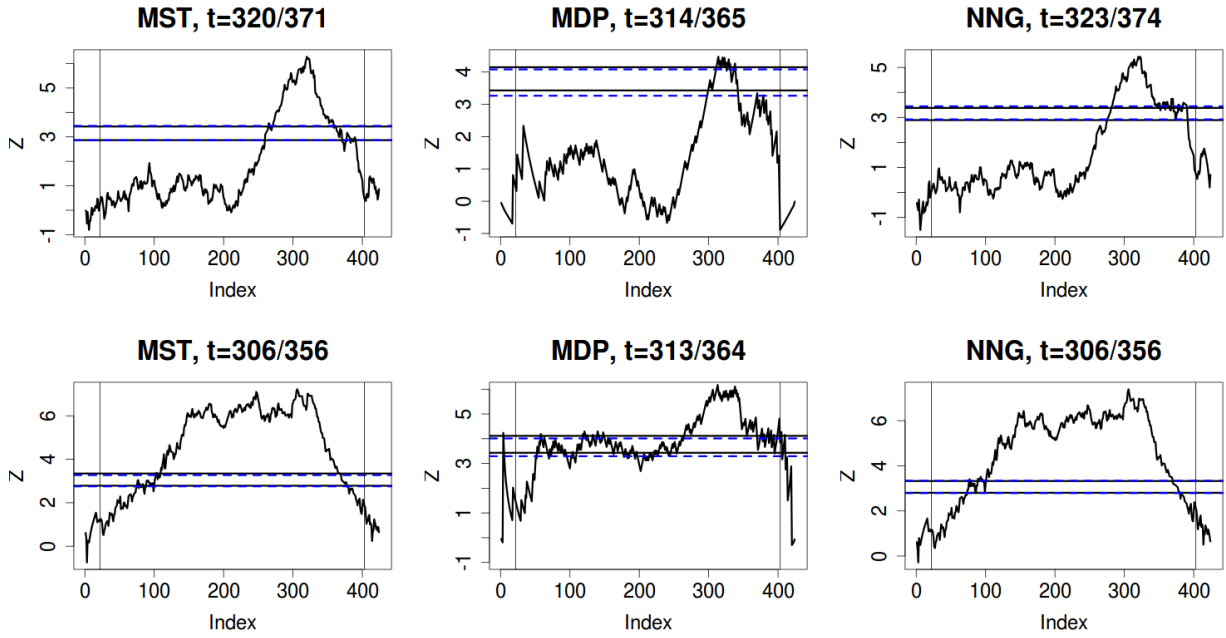

FIG 8. Block permutation results for the authorship data with block size 2.
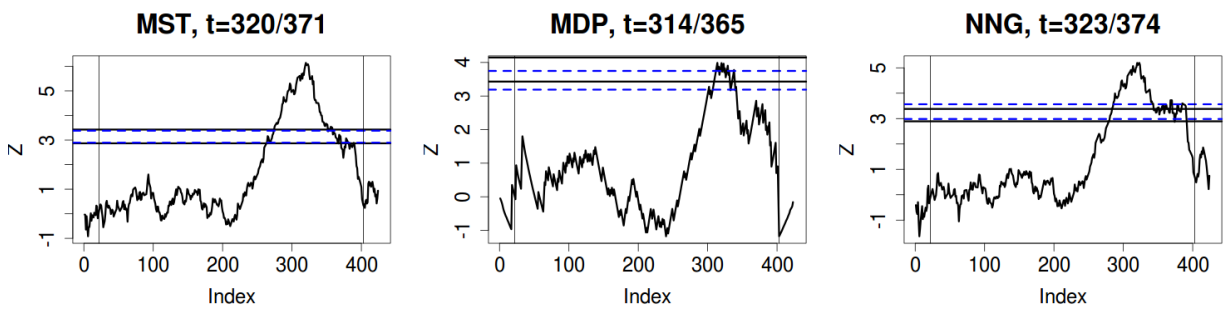

MST, $\mathrm{t}=306 / 356$
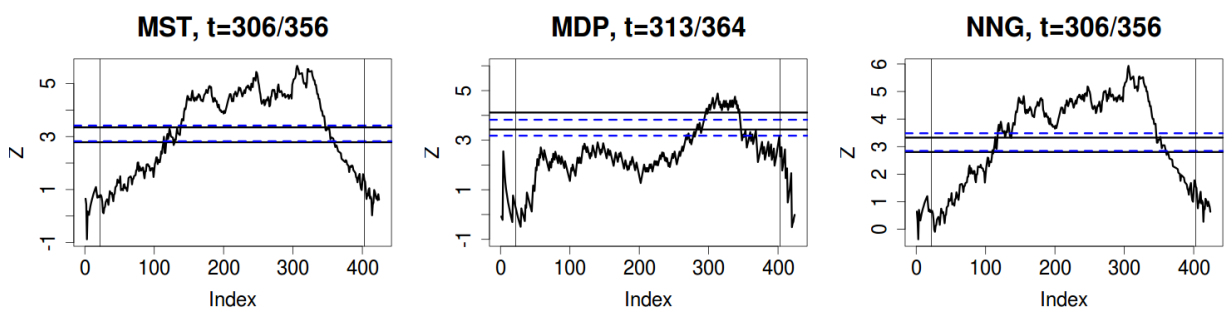

FIG 9. Block permutation results for the authorship data with block size 10. 
D.1.2. Scan over the First 350 Chapters. Here, we show results under block permutations for the authorship data but only using data from the first 350 chapters.

TABLE 8

p-values from 10,000 block permutations for the authorship data only using data from the firt 350 chapters.

\begin{tabular}{|c||ccc|ccc|}
\hline \multirow{2}{*}{ block size } & \multicolumn{3}{|c|}{ Word length } & \multicolumn{3}{c|}{ Context-free word frequency } \\
\cline { 2 - 7 } & MST & MDP & NNG & MST & MDP & NNG \\
\hline \hline 1 & 0.0485 & 0.1079 & 0.3053 & 0 & 0.0019 & 0 \\
\hline 2 & 0.0918 & 0.1345 & 0.4287 & 0 & 0.0033 & 0 \\
\hline 5 & 0.1838 & 0.1788 & 0.5490 & 0 & 0.0029 & 0 \\
\hline 10 & 0.2330 & 0.2335 & 0.6360 & 0 & 0.0127 & 0 \\
\hline
\end{tabular}
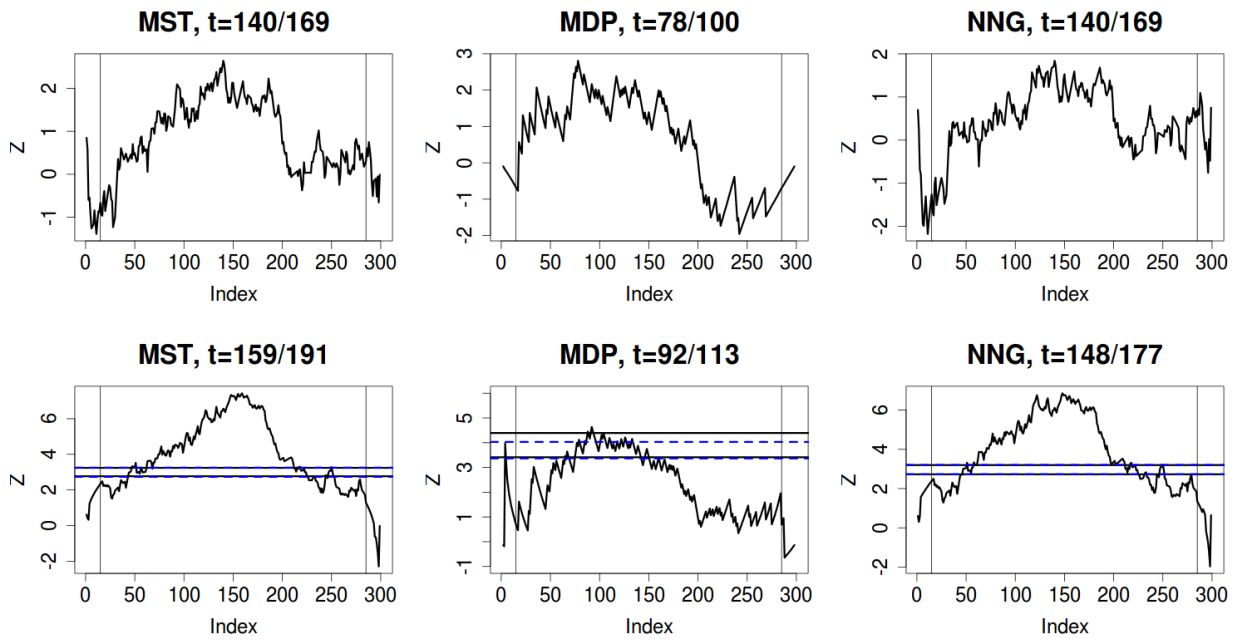

FIG 10. Block permutation results for the authorship data only using the first 350 chapters with block size 2. 

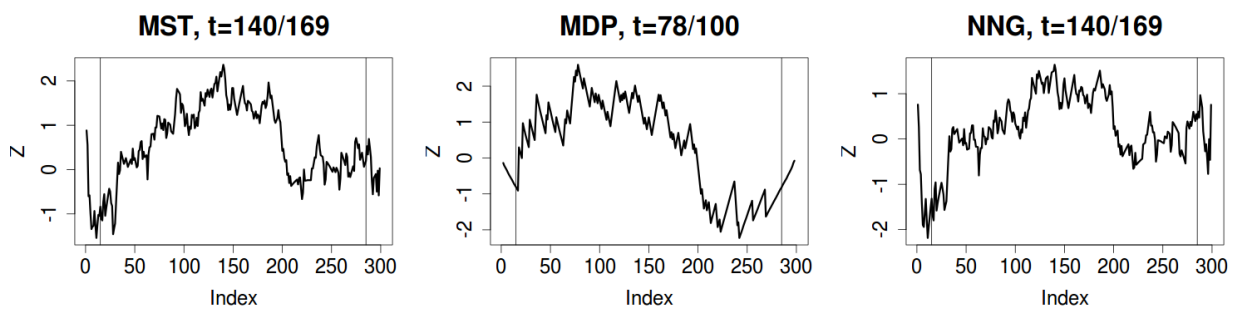

MST, $\mathrm{t}=159 / 191$
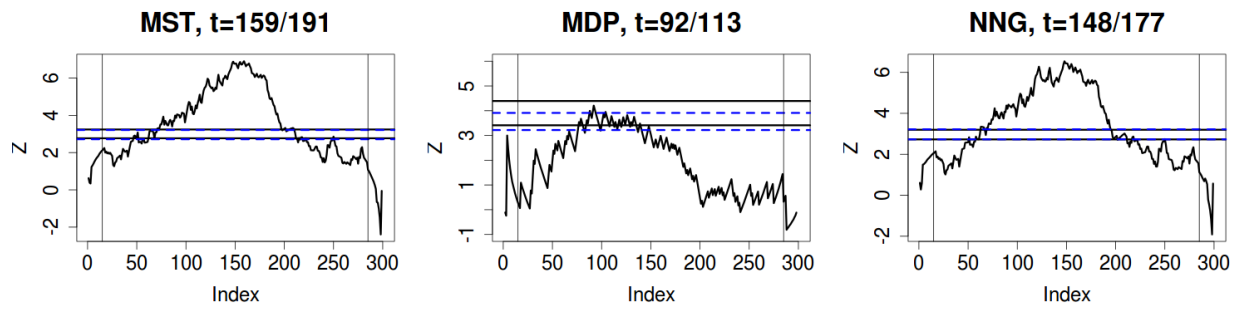

FIG 11. Block permutation results for the authorship data only using the first 350 chapters with block size 5.
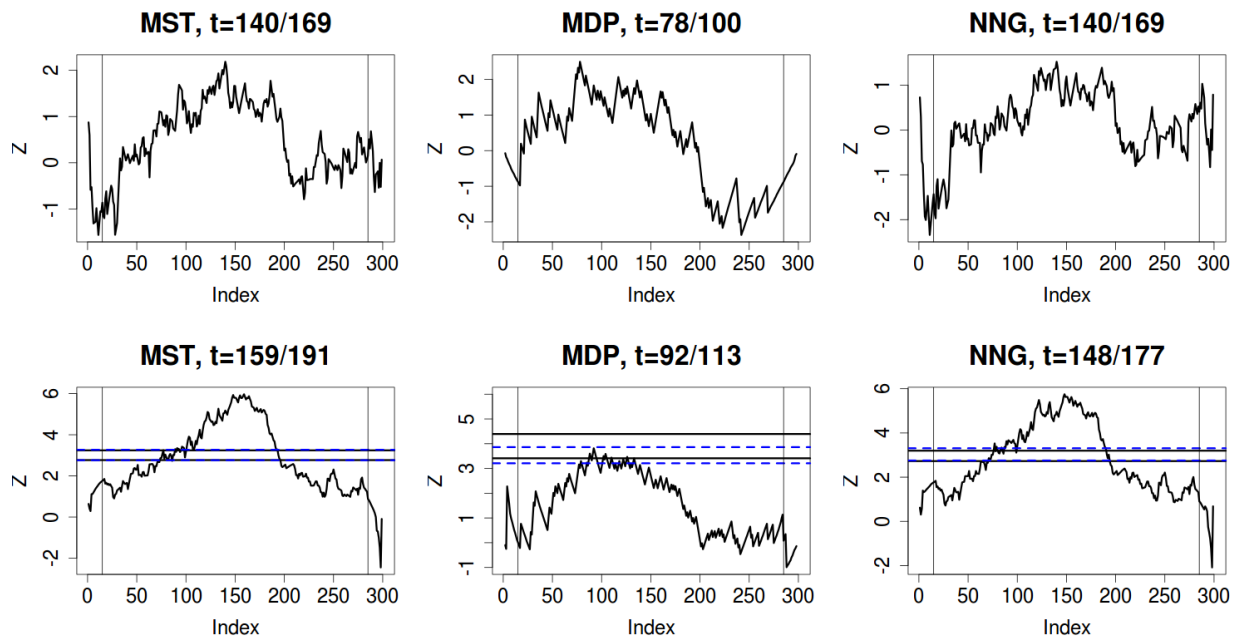

FIG 12. Block permutation results for the authorship data only using the first 350 chapters with block size 10. 
D.2. Friendship Network. Here, we show results under block permutations for the phone call network data.

TABLE 9

p-values from 10,000 block permutations for the phone call network data.

\begin{tabular}{|c||ccc|ccc|}
\hline \multirow{2}{*}{ block size } & \multicolumn{3}{|c|}{ Word length } & \multicolumn{3}{c|}{ Context-free word frequency } \\
\cline { 2 - 7 } & MST & MDP & NNG & MST & MDP & NNG \\
\hline \hline 1 & 0 & 0 & 0 & 0 & 0 & 0 \\
\hline 2 & 0 & 0 & 0 & 0 & 0 & 0 \\
\hline 5 & 0 & 0 & 0 & 0 & 0 & 0 \\
\hline 10 & 0 & 0 & 0.0017 & 0 & 0 & 0 \\
\hline
\end{tabular}
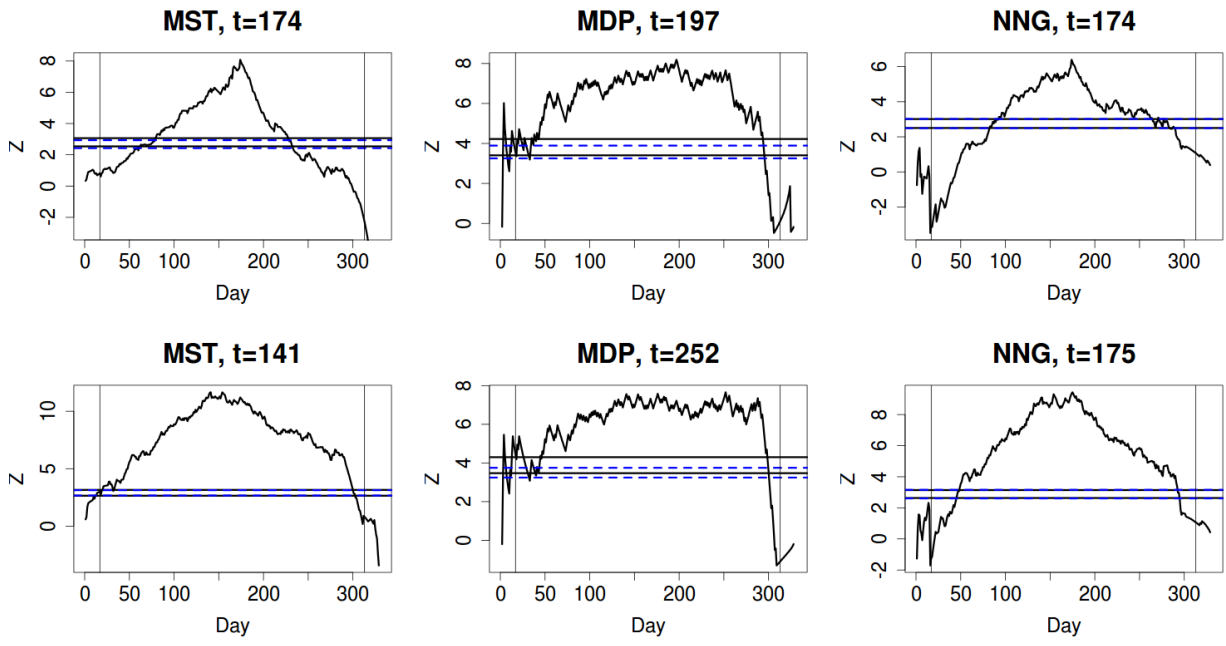

FIG 13. Block permutation results for the phone call network data with block size 2. 

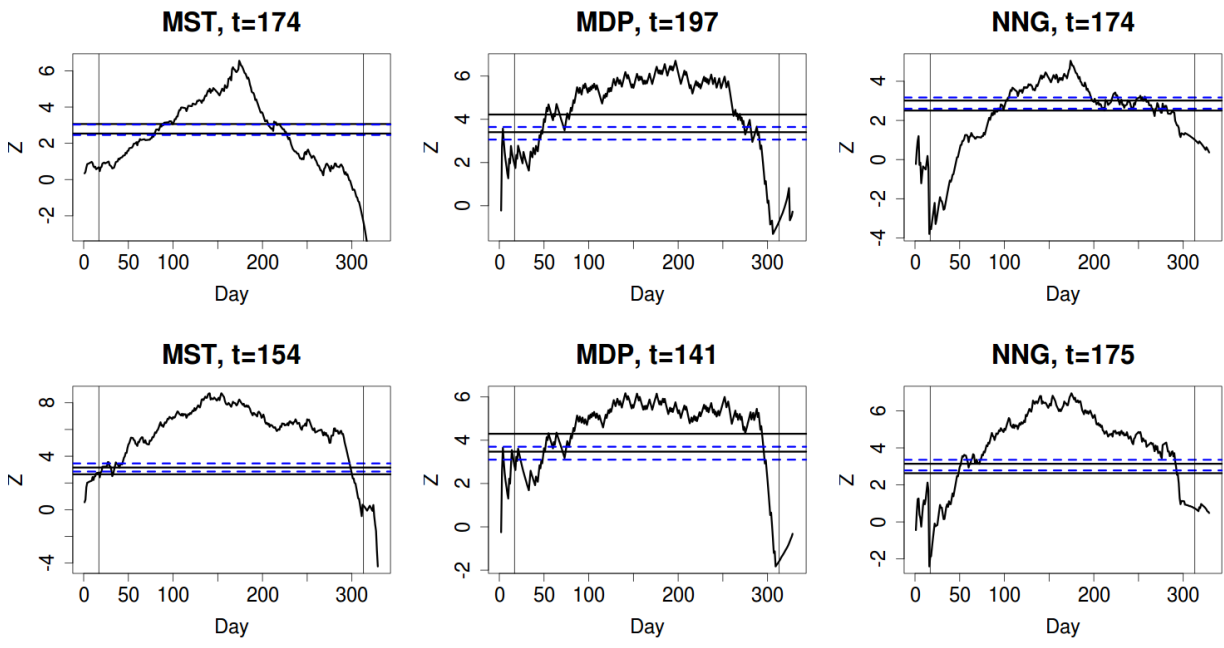

FIG 14. Block permutation results for the phone call network data with block size 5 .
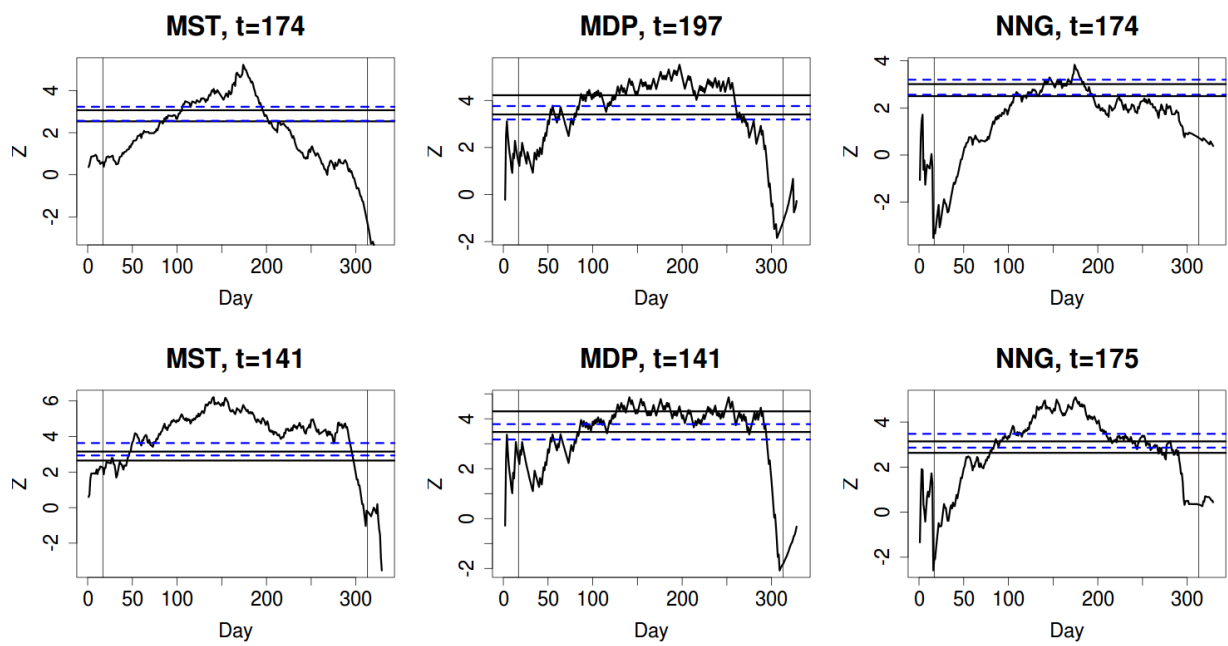

FIG 15. Block permutation results for the phone call network data with block size 10. 


\section{REFERENCES}

Chen, L. H. Y. and Shao, Q. M. (2005). Stein's method for normal approximation. An introduction to Stein's method 4 1-59.

Siegmund, D. (1988). Approximate tail probabilities for the maxima of some random fields. The Annals of Probability 487-501.

Siegmund, D. O. (1992). Tail approximations for maxima of random fields. In Probability theory: proceedings of the 1989 Singapore probability Conference held at the National University of Singapore, June 8-16, 1989 147. Walter de Gruyter.

Woodroofe, M. (1976). Frequentist properties of Bayesian sequential tests. Biometrika $63101-110$.

Woodroofe, M. (1978). Large deviations of likelihood ratio statistics with applications to sequential testing. The Annals of Statistics $72-84$.

Department of Statistics

University of CALIFORNiA, DAVIS

One Shields Avenue

DAVIS, CA 95616

USA

E-MAIL: hxchen@ucdavis.edu
Department of Statistics

The Wharton School

University of Pennsylvania

PhILADELPHIA, PA 19104

USA

E-MAIL: nzh@wharton.upenn.edu 